\title{
Functional Specificity of Callosal Connections in Tree Shrew Striate Cortex
}

\author{
William H. Bosking, ${ }^{1}$ Robert Kretz, ${ }^{2}$ Michele L. Pucak, ${ }^{1}$ David Fitzpatrick ${ }^{1}$ \\ ${ }^{1}$ Department of Neurobiology, Duke University Medical Center, Durham, North Carolina 27710, and Department of \\ Anatomy, University of Fribourg, CH-1700 Fribourg, Switzerland
}

Although callosal connections have been shown to link extensive regions of primary visual cortex, the distribution of these connections with respect to the map of visual space and the map of orientation preference remains unclear. Here we combine optical imaging of intrinsic signals with injection of fluorescent microspheres to assess the functional specificity of callosal connections in the tree shrew. By imaging both hemispheres simultaneously while presenting a series of spatially restricted stimuli, we find that a substantial region of visual space is represented bilaterally. Each hemisphere includes a representation of the ipsilateral visual field that is highly compressed relative to that of the contralateral visual field and is most extensive in the lower visual field, where $\sim 30^{\circ}$ of central visual space are represented bilaterally. Callosal connections extend throughout the region of bilateral representation but terminate in a spatially restricted manner that links visuotopically corresponding sites in the two hemispheres. In contrast, callosal connections appear to terminate without regard for the map of orientation preference, showing little sign of the orientation-specific modular and axial specificity that is characteristic of long-range horizontal connections. By coordinating the activity in the two hemispheres in a way that preserves nearest neighbor relationships, callosal connections may best be viewed as elements of local circuits that operate within a single bilateral representation of visual space.

Key words: optical imaging; intrinsic signals; corpus callosum; horizontal connections; visual field; visual cortex; orientation selectivity; visuotopic; ipsilateral visual field
Unlike the representation of other regions of visual space, the cortical representation of regions near the visual midline is divided between the two hemispheres. Because of this division, callosal connections, which link together sites in primary visual cortex (V1) of the two hemispheres, are thought to play an important role in processing information from the central part of visual space. Compared to other systems of cortical connections, however, there is still considerable uncertainty about how the anatomical arrangement of callosal connections relates to the functional architecture of V1.

Based on early anatomical and physiological studies, callosal connections were thought to be largely confined to the V1/V2 border and to link together neurons whose receptive fields were restricted to regions adjacent to the vertical meridian (VM) (Choudhury et al., 1965; Hubel and Wiesel, 1967; Berlucchi and Rizzolatti, 1968). The limited extent of the connections fit the view that callosal connections served the role of local intrinsic circuits elsewhere in V1: in particular, these connections were thought to supply information from the ipsilateral visual field for cells whose receptive fields spanned the VM (Hubel and Wiesel, 1967). However, although this restricted organization may be the rule for higher primates, in some primates and in many nonprimate species, callosal connections are far more expansive, originating and terminating at sites located up to 2-3 mm away from the V1/V2 border (Swadlow et al., 1978; Cusick et al., 1984, 1985; Sesma et al., 1984; Pritzel et al., 1988; Kretz and Rager, 1990;

Received Oct. 7, 1999; revised Jan. 10, 2000; accepted Jan. 10, 2000.

This work was supported by National Eye Institute Grant EY06821, Swiss National Science Foundation Grant 31-49772.96, and a McKnight Investigator Award (D.F.).

Correspondence should be addressed to David Fitzpatrick, Box 3209, Duke University Medical Center, Durham, NC 27710. E-mail: fitzpat@neuro.duke.edu. Copyright (C) 2000 Society for Neuroscience $0270-6474 / 00 / 202346-14 \$ 15.00 / 0$
Payne, 1991; Payne and Siwek, 1991; Grigonis et al., 1992). The overall extent of callosal connectivity in these species raises the possibility that callosal connections link sites whose receptive fields are significantly displaced from the VM and suggests that the callosal pathway may have more in common with the system of long-distance horizontal connections that exists within a single hemisphere (Rockland and Lund, 1982; Gilbert and Wiesel, 1983). Indeed, evidence that callosal connections and horizontal connections share other features in common, such as laminar distribution, a patchy termination pattern, and the tendency to link sites with similar orientation preference, has led several authors to suggest that callosal connections and horizontal connections might be organized according to similar principles and have similar functions (Innocenti, 1986; Kennedy et al., 1986, 1991; Houzel et al., 1994; Schmidt et al., 1997).

There are, however, reasons to question the notion of a common organizational scheme for callosal and horizontal connections. First, despite their extension away from the V1/V2 border, it has been suggested that callosal connections may link visuotopically corresponding sites in the two hemispheres, rather than sites whose receptive fields are widely displaced (Olavarria, 1996a). This hypothesis is based on physiological evidence from nonprimate species, indicating that the representation of visual space in each hemisphere extends beyond the VM to include a substantial portion of the ipsilateral visual field (Hubel and Wiesel, 1967; Hughes and Vaney, 1982; Whitteridge and Clarke, 1982; Pettigrew et al., 1984; Payne, 1990; Sereno et al., 1991; White et al., 1999) and anatomical evidence for a lack of mirrorsymmetry in the topography of callosal connections (Pritzel et al., 1988; Kretz and Rager, 1990; Olavarria, 1996a). Second, evidence supporting the orientation specificity of callosal connections is far from definitive. The most direct test of this relationship, the 
analysis of the relation between anatomical connections and functional maps, has been explored in only one study (Schmidt et al., 1997). Furthermore, this analysis was performed in strabismic animals, and it is not clear to what extent lack of correlated input from the two eyes may have altered the normal pattern of connections.

In this study, we have used optical imaging techniques in tree shrews to define the maps of visual space and orientation preference in V1 of both hemispheres. We then used anatomical tracing techniques to evaluate both the visuotopic and orientation specificity of callosal connections. Our analysis reveals a substantial representation of the ipsilateral visual field within V1 such that a large part of the binocular region of visual space is represented bilaterally. Callosal connections extend throughout the region of bilateral representation, but they terminate in a spatially restricted manner that links visuotopically corresponding sites in the two hemispheres. In addition, callosal connections appear to lack the orientation specificity that characterizes long-range horizontal connections. Taken together, the properties of callosal connections appear to resemble those of the local component of intrinsic connections within $\mathrm{V} 1$, rather than long-distance connections.

\section{MATERIALS AND METHODS}

Animal preparation. Thirty-one juvenile and adult tree shrews were used for these experiments. Animals were of both sexes and were between $\sim 2$ and 6 months old. No differences in the pattern of intrinsic signals measured was noticed between animals of different ages or sexes, but the best signal-to-noise ratio for position preference experiments was obtained from animals of $\sim 2$ months of age. Anesthesia was induced with a mixture of ketamine hydrochloride $(200 \mathrm{mg} / \mathrm{kg})$ and xylazine (4.7 $\mathrm{mg} / \mathrm{kg})$ given by intramuscular injection. Atropine sulfate $(0.08 \mathrm{mg})$ was given subcutaneously to reduce secretions. Surgical levels of anesthesia were maintained with a 2:1 mixture of $\mathrm{N}_{2} \mathrm{O}: \mathrm{O}_{2}$ and $2 \%$ isoflurane. Body temperature was maintained near $38^{\circ} \mathrm{C}$ with a thermostatically controlled heating blanket. Animals were placed in a stereotaxic frame, an incision was made in the scalp, muscle and fascia were reflected bilaterally, and the bone overlying visual cortex was thinned by scraping with a fine scalpel. All wound margins were treated with a long-lasting local anesthetic (bupivacaine), and pressure points were treated with lidocaine ointment. Animals were paralyzed by administration of pancuronium bromide (initial dose $0.4 \mathrm{mg}$ for the first $1-1.5 \mathrm{hr}$, then $0.2 \mathrm{mg} / \mathrm{hr}$ ). Tree shrews were artificially respired at a rate and volume sufficient to maintain expired $\mathrm{CO}_{2}$ at 3-4\%. During optical imaging, isoflurane levels were reduced to $0.5 \%$ and the $\mathrm{N}_{2} \mathrm{O}: \mathrm{O}_{2}$ mixture to $1: 1$. Any signs of distress evident in the electrocardiogram or expired $\mathrm{CO}_{2}$ were treated by immediately increasing the level of isoflurane.

Zero power contact lenses were used to protect the corneas, and stimulus focus was assessed by viewing the retina through an ophthalmoscope held at screen distance from the eye. In all animals examined, the retinal vasculature was in sharp focus with little or no correction used on the ophthalmoscope, and no additional corrective lenses were used. The location of the optic disk for each eye was projected onto a screen in front of the animal. Under our anesthesia and paralysis conditions, the optic disk projections were found to lie in a very stereotyped and symmetrical position with respect to the animal midline. The optic disk projection for the left eye was located $51.4 \pm 3^{\circ}$ from the animal midline and $6.4 \pm 1.6^{\circ}$ above eye height, and the optic disk projection for the right eye was located $50.5 \pm 2.3^{\circ}$ from the animal midline and $6.7 \pm 2.3^{\circ}$ above eye height (mean $\pm \mathrm{SD} ; n=12$ animals). Proper eye alignment was confirmed by electrophysiology (see Results). Apparent shifts in eye position during $12 \mathrm{hr}$ or longer recording sessions were small relative to the receptive field size of cortical neurons: $1.8 \pm 1.7^{\circ}$ for absolute change in azimuth for left eye, $1.6 \pm 1.4^{\circ}$ for absolute change in azimuth for the right eye (mean $\pm \mathrm{SD} ; n=5$ animals).

Optical imaging. Optical imaging of intrinsic signals was accomplished using an enhanced video acquisition system (Optical Imaging, Inc.) using techniques similar to those we have described previously (Bosking et al., 1997). Images of the surface of left and right visual cortex were acquired simultaneously directly through the thinned bone. For all animals, red light $(700 \pm 10 \mathrm{~nm})$ was used to illuminate the cortical surface to help reduce the presence of vascular artifacts. In addition, for some animals, artifacts caused by large changes in blood flow and oxygenation in the central sinus area were reduced by using an opaque red marker to mask out portions of this region of bone.

Visual stimulation for optical imaging was provided by a separate stimulus computer (386 PC with SGT+ graphics board and STIM software provided by Kaare Christian). The stimulus monitor was placed $28.6 \mathrm{~cm}$ in front of the animal and subtended $80^{\circ}$ of azimuth and $60^{\circ}$ of elevation at this distance. All stimuli were presented at a display rate of $60 \mathrm{frames} / \mathrm{sec}$. Stimuli for orientation preference experiments consisted of high-contrast rectangular wave gratings $\left(3^{\circ}\right.$ dark phase, $1^{\circ}$ light phase, drifted at $7.5 \% \mathrm{sec}$ ) presented at four different orientations. Each grating was moved back and forth along an axis orthogonal to its orientation. A single trial consisted of presentation of one orientation for $9 \mathrm{sec}$, with acquisition of video images during the last $8 \mathrm{sec}$, an interstimulus interval of $8 \mathrm{sec}$, and then presentation of the orthogonal orientation, again with data acquisition during the last $8 \mathrm{sec}$. Video images were acquired at a rate of $30 \mathrm{frames} / \mathrm{sec}$, but all frames acquired during the $8 \mathrm{sec}$ period were summed together before further analysis. Between 8 and 20 trials were used for each pair of orientations $\left(0 / 90^{\circ}, 45 / 135^{\circ}\right)$. Difference images of orientation preference were obtained by subtracting data acquired during presentation of one orientation from data acquired during presentation of the orthogonal orientation.

The stimulus used for position preference experiments was a highcontrast vertical bar $\left(0.5^{\circ}\right.$ in width, $60^{\circ}$ in length $)$, panned back and forth within a $2^{\circ}$ wide window for $9 \mathrm{sec}$. Video images were acquired at a rate of $30 \mathrm{frames} / \mathrm{sec}$ during the last $8 \mathrm{sec}$ of this period. All data acquired during presentation of a blank screen were subtracted from data acquired during presentation of the vertical bar stimulus to obtain an image reflecting the cortical activity in response to the vertical bar. Each experiment consisted of $\sim 8-20$ trials with the stimulus located in the same position. In separate experiments, we tested the response of the animal with this stimulus placed in locations ranging from $16^{\circ}$ left of center of the monitor to $16^{\circ}$ right of center.

During imaging experiments, we determined the position of the VM using one of two methods. First, the VM was operationally defined as the screen location that gave the most symmetrical activation of the left and right cortex. It was usually possible to determine this position within $\sim 1-2^{\circ}$. In some animals, a separate determination of the position of the VM was obtained by projecting the position of the optic disks onto a screen in front of the animal and calculating the position of a line halfway between the two disks. On average, the difference in location of the VM determined by these two methods was small $\left(1.1 \pm 1.2^{\circ}\right.$; mean $\pm \mathrm{SD} ; n=$ 12 ), and throughout the paper we use the VM determined by symmetrical activation as the basis for presentations and calculations.

Electrophysiology. In some animals, a small hole was opened in the skull over V1 after optical imaging. Tungsten electrodes, $50 \mathrm{~mm}$ in length, $14 \mathrm{M} \Omega$ in resistance (FHC, Bowdoinham, ME), were inserted through the dura mater and were used to record multiunit responses. The position of recording locations relative to the surface vasculature was noted. Multiunit aggregate receptive fields were plotted using a computer-assisted minimum response technique. In most cases, we considered both the spikes of individual units and the general "hash" response in determining the borders of the receptive fields. When possible, we plotted the receptive field for both left and right eye responses.

Injection of fluorescent microspheres. In eight animals in which we performed optical imaging and in one additional animal in which we did not perform optical imaging, we made pressure injections of latex microspheres conjugated to either rhodamine (red beads) or fluorescein (green beads) using a Picospritzer (beads provided by Dr. L. Katz, Duke University). The number and timing of pulses used to obtain a reasonable size injection was variable, but the general strategy was to lower the tip to a depth of $700 \mu \mathrm{m}$, give three to six pulses, and then repeat this procedure at depths of $600,500,400,300,200$, and $100 \mu \mathrm{m}$. Each pulse was $\sim 5 \mathrm{msec}$ in duration at a pressure of $25 \mathrm{psi}$.

Histology. At the conclusion of optical imaging, or after a $3 \mathrm{~d}$ survival period for animals in which we made bead injections, animals were transcardially perfused with $0.9 \%$ saline followed by $4 \%$ paraformaldehyde in $0.1 \mathrm{M}$ phosphate buffer and then $10 \%$ sucrose in $0.1 \mathrm{M}$ phosphate buffer. Brains were removed and placed in $20 \%$ sucrose in $0.1 \mathrm{M}$ phosphate buffer. Visual cortex from each hemisphere was removed and flattened between slides overnight in $0.1 \mathrm{M}$ phosphate buffer containing $20 \%$ sucrose. Tangential sections $40 \mu \mathrm{m}$ in width were cut on a freezing microtome. The first one or two sections were often cut at 60 or $80 \mu \mathrm{m}$ 
to ensure that a large portion of the surface vasculature over V1 was captured in one section. The distribution of bead-labeled cells in the cortex was plotted by hand using a camera lucida or with the assistance of a computerized plotting system (Neurolucida; Microbrightfield, Colchester, VT). Hand-plotted sections were scanned into a computer for alignment with optical imaging data. After plotting of bead-labeled sections, or in animals without bead injections, Nissl stains were performed on the cortical sections.

Alignment of Nissl-stained tissue and bead-labeled cell distributions with optical imaging data were performed using a modified version of the public domain program NIH Image (original version developed at the National Institutes of Health, available on the Internet at http: $\mid r s b . i n f o$. nih.gov/nih-image/; see Bosking et al., 1997 for details of modification). Briefly, scanned images or text files containing data plotted with the Neurolucida system were read into memory and aligned to the reference image taken during the optical imaging phase of the experiment. Surface and radial blood vessels were the primary landmarks used to align section to section and to align sections to the reference image. For the experiments presented in this report, we used alignment routines that allowed not only global scaling, rotation, and translation of the camera lucida or Neurolucida drawings, but also differential $x$-axis and $y$-axis scaling and the capability to induce a defined amount of curvature to the drawing to help correct for uneven flattening or shrinkage. These additions enabled slightly more accurate alignments on plots that spanned large portions of V1.

Analysis. Images obtained by optical imaging were $655 \times 480$ pixels in resolution, with $\sim 62$ pixels $/ \mathrm{mm}$ as a result of the lens combination used. High-frequency noise was removed from orientation difference images by using a mean filter kernel between $5 \times 5$ and $10 \times 10$ pixels in size. Low-frequency noise in the orientation difference images was reduced by convolving the image with a $50 \times 50$ pixel mean filter kernel and subtracting the result from the original image. Difference images were normalized by dividing the deviation from the mean at each pixel by the average absolute deviation across the entire image (Weliky et al., 1995). Finally, vector summation of the difference images was done on a pixel by pixel basis to create a color-coded orientation preference map (Bonhoeffer and Grinvald, 1991, 1993; Blasdel, 1992).

The only filtering applied to the imaging data for position specificity shown in Figures 6 and 7 was a mean filter $5 \times 5$ or $7 \times 7$ pixels in size.
To help reduce vascular artifacts in the case presented in Figures 3 and 4 , we used the reference image to create a mask indicating the location of the major blood vessels in the imaging field of view. This mask was used to selectively filter the raw data from each image in the position series. The grayscale value for each pixel in the data images that was located in the blood vessel mask was replaced by the mean of the grayscale values of the surrounding pixels. To calculate the mean for each pixel, we used the minimum area possible while requiring a minimum of 160 pixels that were not in the blood vessel mask. Grayscale values for those pixels that were not in the mask were not changed during this filtering. In the region of V1 that we imaged, this process altered only $17 \%$ of the pixel values. We then mean filtered each image using a $7 \times$ 7 kernel and replaced each frame in the original position series of 17 frames with the average of that frame and the two adjacent frames to obtain the series of 15 images that are displayed in Figure 3.

The position preference map shown in Figure 4 was obtained by combining data from the 15 images shown in Figure 3. For each pixel, a position tuning curve was constructed by obtaining the grayscale response value for each position tested. Then a Gaussian curve was fit to this data, and the location of the peak of the Gaussian was taken as the preferred position for that site. The equation used for this process was:

$$
\text { Pixel value }=\mathrm{p} 1+\mathrm{p} 2 * e^{-((x-\mathrm{p} 3) / \mathrm{p} 4)^{2}}
$$

where $p 1, p 2, p 3$, and $p 4$ are parameters specifying the shape and location of the Gaussian curve and $x$ refers to the horizontal position of the stimulus on the screen. The initial values for the parameters were established from the minimum and maximum pixel values in the raw data for that location as follows: $p 1=$ minimum pixel value, $p 2=$ maximumminimum pixel value, $p 3=$ undetermined, and $p 4=2$. Using these initial values, the location of the peak ( $p 3)$ was allowed to vary in steps of 0.1 , and the location resulting in the smallest error was recorded. This value for $\mathrm{p} 3$ and the values listed above for the other parameters were then fed to an iterative algorithm that allowed all four parameters to vary. The algorithm implemented was a version of the downhill simplex method (Press, 1992). The final value of $\mathrm{p} 3$ after this iterative search was taken as the preferred position of the site in question, and the position preference map is then a simple color coding of the position preference across the sampled region of visual cortex. Limits were set for each
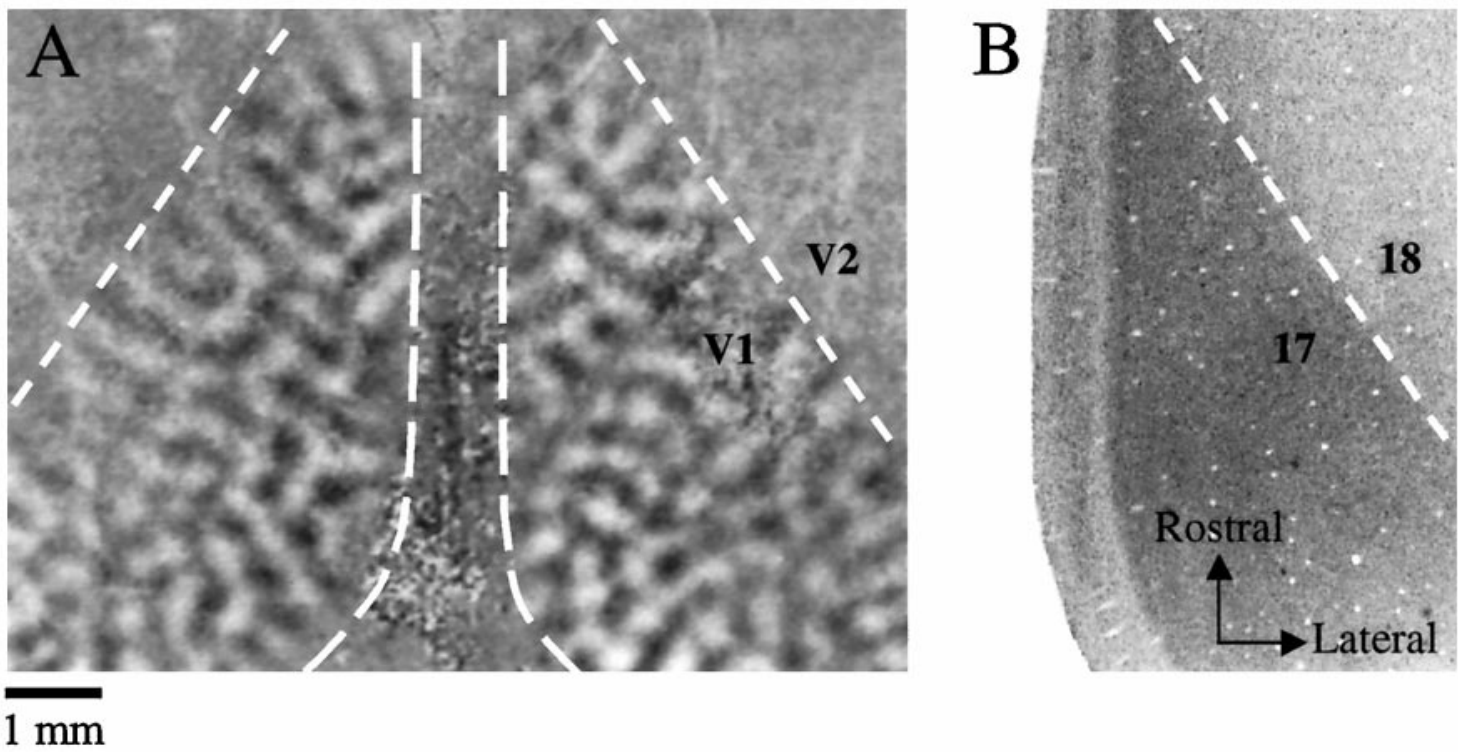

Figure 1. V1/V2 border defined by optical imaging and Nissl staining (animal TS9756). A, Bilateral pattern of orientation selectivity demonstrated by optical imaging. The midline of the animal is in the center of the image, and rostral is toward the top of the page. The dashed lines indicate the portion of the imaged area corresponding to V1 in each hemisphere. All optical imaging data for the remainder of the paper will be presented in this orientation. Dark areas of V1 were strongly activated by a grating oriented at $45^{\circ}$; white areas were strongly activated by a grating oriented at $135^{\circ}$. Strong orientation signal is visible throughout V1 on each side, but not in V2. The dashed line in right visual cortex indicating the location of the V1/V2 border was defined by the Nissl-stained section shown in $B$. The dashed line in left visual cortex indicating the V1/V2 border was drawn directly from the orientation difference image. $B$, Photomicrograph of a Nissl-stained tangential section through V1. This section was aligned to the optical imaging reference image using techniques described in Materials and Methods and corresponds to the right side of the image shown in $A$. The darkly stained region of the image corresponds to area 17, and the dashed line is placed at the border of the lightly stained and darkly stained regions. Scale bar applies to both figures. 

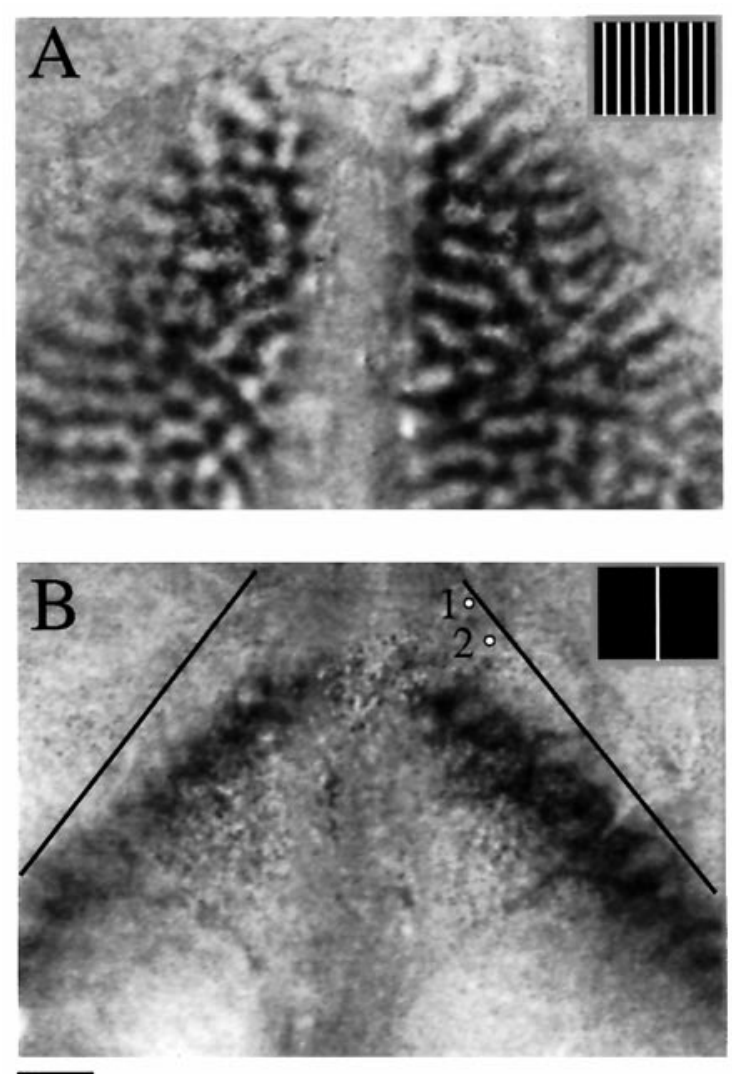

$1 \mathrm{~mm}$

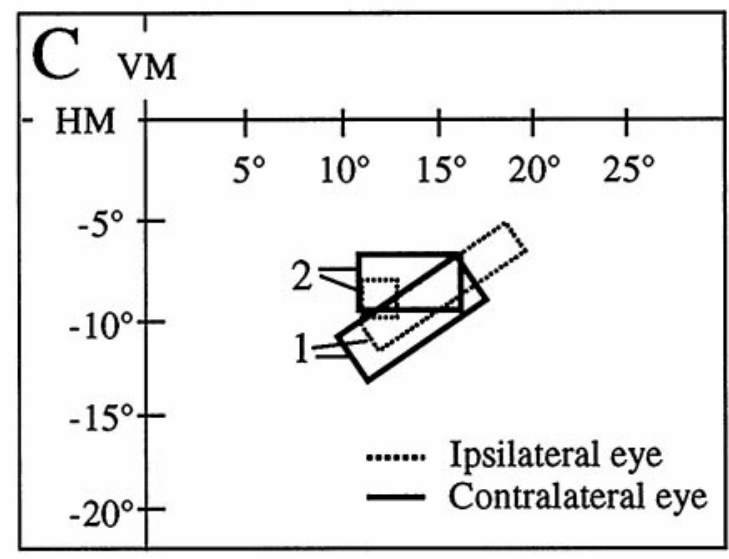

Figure 2. Bilateral orientation difference signal and bilateral response to a stimulus at the VM (animal TS9751). A, Bilateral orientation difference signal. Dark areas of the image were preferentially activated by a vertical grating (stimulus shown in inset), and white areas were preferentially activated by a horizontal grating. $B$, Bilateral pattern of activity in response to a bar stimulus $\left(0.5^{\circ}\right.$ wide moved in a $2^{\circ}$ wide window placed at the VM, shown in inset). Dark areas of the image were strongly activated by the bar stimulus as compared to a blank screen. The thin black lines denote the V1/V2 border as defined by the orientation signal shown in $A$. Note that the cortical representation of the VM is displaced from the V1/V2 border in each hemisphere, implying the existence of a representation of the ipsilateral visual field. Scale bar applies to $A$ and $B$. $C$, Multiunit receptive field plots for the two recording sites depicted in $B$. The ipsilateral (dashed lines) and contralateral eye (thick lines) receptive fields are overlapping at each location, indicating minimal misalignment of the eyes. The receptive fields are located in the right (ipsilateral) visual field, consistent with the optical imaging results. parameter for the Gaussian fitting process, and any pixel in the position preference map where the limits were exceeded was replaced by the mean of the surrounding area. Again we used the minimum radius possible to obtain the mean value, but in this case we required only 50 pixels for averaging. The final position preference map was smoothed with a $7 \times 7$ pixel mean filter.

Selectivity of labeled cell distributions. After alignment of the histological sections to the optical imaging data, we quantified the orientation selectivity of the labeled cell distributions by counting the number of cells that were found over different regions of the orientation preference map within $10^{\circ}$ bins of orientation preference. For intrinsic cell distributions we counted only the cells that were $>500 \mu \mathrm{m}$ from the injection site. Cells that were located in V2 were excluded from the analysis.

\section{RESULTS}

Our results are grouped into four sections. In the first section, we describe the map of visual space revealed by optical imaging and compare this map to the map of orientation preference and the histologically defined area 17/area 18 border. In the second section, we present electrophysiological experiments that confirm the existence of an ipsilateral visual field representation within V1. Finally, in the third and fourth sections, we present the results of experiments in which we combined optical imaging with injection of fluorescent beads to assess visuotopic and modular specificity of callosal connections.

\section{Ipsilateral visual field representation revealed by optical imaging}

Optical imaging was performed directly through the thinned bone over left and right visual cortex simultaneously. We obtained robust patterns of differential activity related to orientation preference from a large region of the exposed cortex of both hemispheres (Fig. 1 $A$ ). We have previously described some of the prominent features of the orientation preference map in the tree shrew (Bosking et al., 1997). Interestingly, bilateral imaging of orientation preference allowed us to determine that the pattern of activity associated with a particular orientation was not identical, or mirror symmetric, in the two hemispheres (Fig. $1 A$ ). In addition, the area of cortex that provided strong orientation difference signals provided a convenient way to define the V1/V2 border in our optical imaging experiments. In Nissl-stained sections of tree shrew visual cortex, the area $17 / 18$ border is clearly defined by an abrupt change in staining intensity (Fig. $1 B$ ). This border corresponds to the V1/V2 border defined by electrophysiology (Kaas et al., 1972) and with the limits of the area of cortex providing strong orientation difference signal in optical imaging experiments (Fig. 1A).

To investigate the map of visual space, we compared the pattern of activity elicited by a single bar stimulus, placed in a particular location, to the pattern of activity obtained during presentation of a blank screen (see Materials and Methods for details). This stimulus was selected because it was spatially restricted in one dimension yet was still capable of driving strong cortical activation. The activity elicited by this stimulus was restricted to bands of cortex 1-2 mm in width that were elongated approximately parallel to the V1/V2 border (Fig. 2B). The strength of the signal within these bands was not uniform, and comparison of the activation pattern within each band with orientation difference maps from the same animal revealed that the areas of more intense activation correspond to vertical isoorientation domains (Fig. 2, compare $A, B$; see Fig. 7A, $B$ ).

Presentation of the bar stimulus in the central regions of visual space elicited bands of activity in both hemispheres. Because there was very good agreement between the position of the stimulus that elicited a symmetrical pattern of bilateral activation 


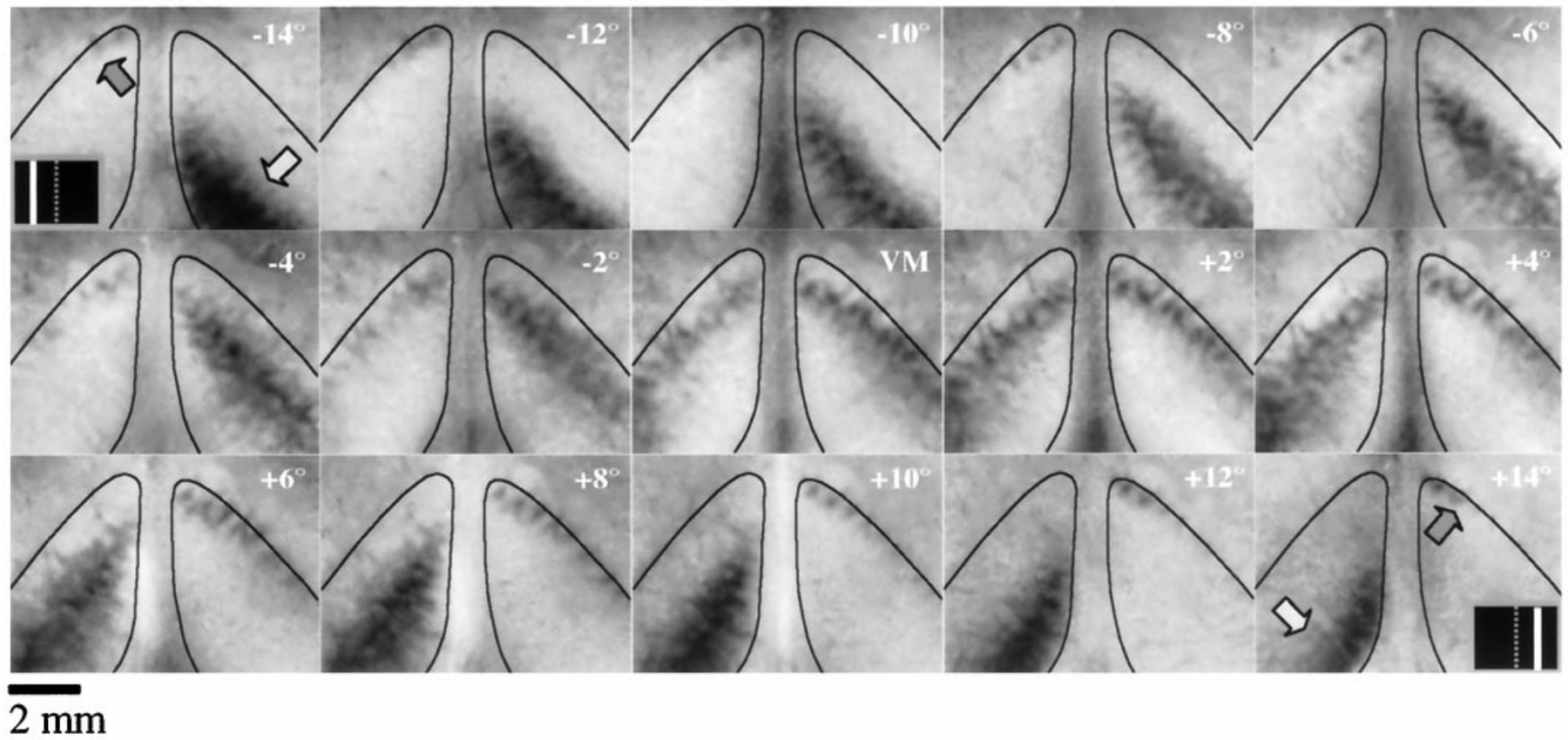

Figure 3. Cortical response to a $0.5^{\circ}$ wide bar stimulus placed in a series of 15 positions from $14^{\circ}$ left of the $\mathrm{VM}\left(-14^{\circ}\right)$ to $14^{\circ}$ right of the $\mathrm{VM}\left(+14^{\circ}\right)$, spaced at $2^{\circ}$ intervals (animal TS9762). The thin black lines in each frame outline V1 as determined by the limits of the area of strong orientation selectivity. Dark areas indicating stimulus-driven cortical activation are seen bilaterally in each image. In the first and last frames of the series, the light gray arrow indicates the location of activated cortex in the contralateral hemisphere, the dark gray arrow indicates the location of activated cortex in the ipsilateral hemisphere, and the inset indicates the location of the stimulus. Every stimulus position except the VM produced a non-mirror-symmetric pattern of cortical activation.

and the midpoint between the two optic disk representations, we opted to use symmetrical activation to define the location of the VM (see Materials and Methods). Comparison of the location of the bands of cortical activation obtained when the stimulus was presented at the VM with the limit of strong orientation signal in the same hemispheres (Fig. $2 A$ ) revealed that these bands were not juxtaposed to the V1/V2 border. Instead, the bands were displaced medially, the displacement being greater in more rostral portions of V1. Assuming that the eyes were properly aligned on the monitor, this observation implies a significant representation of the ipsilateral visual field within V1, a departure from the results of previous physiological mapping studies (Kaas et al., 1972; Kaas, 1980).

We checked for misalignment of the eyes in this animal by making physiological recordings from two sites in the lateral region of $\mathrm{V} 1$ in the right hemisphere (Fig. $2 C$ ). At each recording site, the left and right eye receptive fields were highly overlapping. The receptive fields for these sites were located in the lower visual field, as expected based on previous physiological mapping studies, and were located well into the right (ipsilateral) visual field, as predicted from the imaging results.

Physiological recordings from additional animals $(n=6)$ confirmed that errors in eye alignment could not account for our imaging results. Overlapping ipsilateral and contralateral eye receptive fields were found in all except one animal. In the remaining animal, the eyes were diverged by $\sim 6^{\circ}$; data from this animal are not used for any of the analysis in this paper. To further check for systematic errors in eye alignment that might affect our results, we calculated the difference in the position of receptive field centers of the ipsilateral and contralateral eye receptive fields from all sites for which we had binocular data. The average errors in receptive field alignment were calculated separately for the horizontal axis (dx) and vertical axis (dy) for each animal. The average misalignment measured from the five animals was $\mathrm{dx}=-0.27^{\circ}$ and $\mathrm{dy}=0.73^{\circ}$. These misalignments in receptive field centers are small relative to the size of the average receptive fields of layer $2 / 3$ neurons and cannot account for the substantial representation of the ipsilateral visual field that we found in V1.

To examine the structure of the ipsilateral visual field representation, we tested cortical response to different positions ranging from up to $16^{\circ}$ left to $16^{\circ}$ right of the $\mathrm{VM}$, spaced at $2^{\circ}$ intervals. The responses to fifteen of the stimuli tested for one case are shown in Figure 3; a bilateral pattern of activation was elicited by all 15 of the stimuli shown. The light gray arrows in the first and last frames of the series indicate the position of cortical activation that is contralateral to the stimulus; the dark gray arrows indicate the position of cortical activation that is ipsilateral to the stimulus. Note the non-mirror-symmetric pattern of cortical activation for all stimulus positions except the VM. The locus of activity in the contralateral hemisphere is shifted further away from the V1/V2 border, and the locus of activity in the ipsilateral hemisphere is shifted closer to the V1/V2 border and occupies a much smaller extent. A similar pattern of responses was seen in all animals that were tested $(n=16)$.

Animation of the series of images shown in Figure 3 provides dramatic evidence of the orderly representation of visual space and the smooth progression of responses seen throughout V1. It is also possible to use these images to construct a position preference map that provides the same information about the structure of the map of visual space in a single image. For each site in cortex, the position preference was calculated by fitting a Gaussian curve to a plot of response magnitude versus position number. This curve is essentially a position tuning curve for that location 


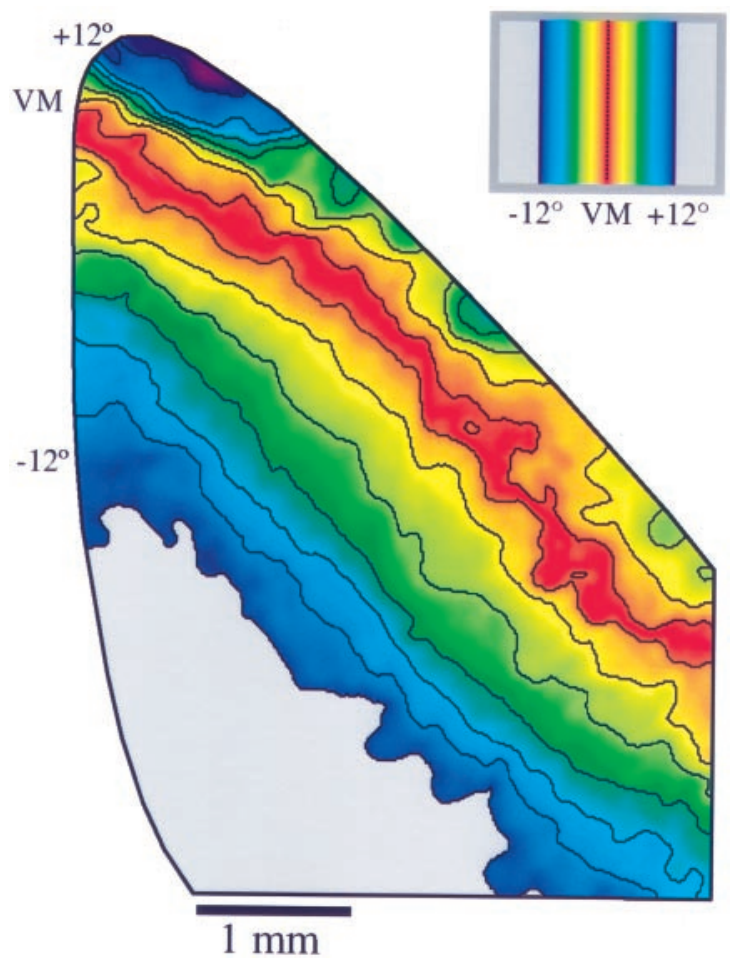

Figure 4. Position preference map for right visual cortex generated from the data shown in Figure 3 (animal TS9762). Position preference was calculated for each pixel location by fitting a Gaussian curve to a plot of the response level ( grayscale value) versus position number (see Materials and Methods for details). Only regions that were within V1 (as determined by the area of strong orientation signal) are depicted. Position preference is color-coded according to the key shown in the inset. Each color represents a particular distance from the VM so that corresponding azimuths in ipsilateral and contralateral visual space are represented by the same color. The black lines indicate $2^{\circ}$ contour intervals in the map of visual space. Note the compression of the ipsilateral visual field representation relative to the contralateral visual field representation.

in cortex, and the peak of the fitted Gaussian curve can be taken as an estimate of the position preference of that site. This procedure is repeated for each pixel in the image. Although this procedure is similar in principle to using vector summation to combine responses from different angle maps to create an orientation preference map, it has the advantage that it can be used to analyze noncyclic features such as position preference.

Figure 4 shows the position preference map for the right hemisphere of the case shown in Figure 3. In this image, position preference is color-coded according to the key shown in the inset. Position preferences are shown only for the portion of the imaging frame that lay in V1. Three important features about the representation of visual space are apparent: (1) There is a smooth progression of position preference across the entire extent of $\mathrm{V} 1$, including the contralateral and ipsilateral visual field representations. (2) The ipsilateral visual field representation is highly compressed relative to the contralateral visual field representation. Within the contralateral visual field representation, $\sim 12^{\circ}$ of visual space are represented within $\sim 2 \mathrm{~mm}$ of cortex. The same amount of the ipsilateral visual field is represented within $\sim 1 \mathrm{~mm}$ of cortex. (3) The amount of the ipsilateral visual field represented varies systematically with elevation, being largest in the representation of the lower visual field (Fig. 4, rostral portion).

\section{Ipsilateral visual field map confirmed by electrophysiology}

A comprehensive examination of the extent of the ipsilateral visual field representation and its relationship to the cytoarchitectonically defined area 17 was performed in one animal using electrophysiology. In Figure $5 A$, the locations of 10 recording sites from this animal are illustrated over a Nissl-stained section. The locations of the recording sites were initially plotted relative to the blood vessel pattern in a reference image taken during an optical imaging experiment. The tissue section was then aligned to the reference image to obtain the position of the recording sites relative to the Nissl-stained section (see Materials and Methods for details). The receptive field locations from these 10 sites are shown in Figure $5 B$. At the first recording site, we confirmed eye alignment by plotting both ipsilateral and contralateral eye receptive fields (thin black and dashed black boxes \#1). At the remaining sites, we plotted only the contralateral eye receptive field. For sites $1-6$, there is a consistent movement of the receptive fields into the ipsilateral visual field with lateral movement of the recording sites. For sites 9 and 10, the progression of receptive field locations has reversed, and there is also a large increase in receptive field size. This implies that the V1/V2 border was between sites 6 and 9 . At site 7, we obtained receptive field plots at two different depths. At one depth the receptive field was small and located well into the ipsilateral visual field, suggesting that the recording site was in V1. At another depth the receptive field was much larger and located closer to the VM, suggesting that the recording site was in V2. Together the evidence suggests that the V1/V2 border was very near sites 7 and 8 , a location that corresponds precisely to the area $17 / 18$ border visualized on the Nissl-stained section that was aligned to the reference image. This confirms an orderly representation of the ipsilateral visual field that extends for $\sim 1 \mathrm{~mm}$ within the limits of the cytoarchitectonically defined area 17 and not within a separate transition zone as described in the cat (Payne, 1990). Note that there was a large jump in receptive field position from well into the ipsilateral visual field to back near the VM as we crossed the border into V2. This result suggests that there is a less extensive representation of the ipsilateral visual field in V2 or that it is smaller and more difficult to detect.

\section{Visuotopic specificity of callosal connections}

The non-mirror-symmetric activation patterns observed in response to individual stimuli bear a striking resemblance to the non-mirror-symmetric pattern of callosal connections that have been previously described in the tree shrew and other species (Pritzel et al., 1988; Kretz and Rager, 1990; Olavarria, 1996a). This observation suggests that callosal connections may link locations in the two hemispheres that respond to the same part of visual space. We tested this idea by combing optical imaging with injection of fluorescently labeled microspheres (beads).

The relation between the pattern of bead labeling and cortical activity patterns was assessed by using the pattern of surface and radial blood vessels to align the anatomical data from tangential sections to the optical imaging data. As shown in Figure $6 \mathrm{~A}$, line drawings of the blood vessels in the first section (yellow lines) were aligned to the reference image taken during the optical imaging phase of the experiment. Figure $6 \mathrm{~B}$ provides a higher magnification view of the alignment of the blood vessels in the first section (yellow) as well as the alignment of the radial vessels (blue) from a deeper section that contained bead labeled cells. 

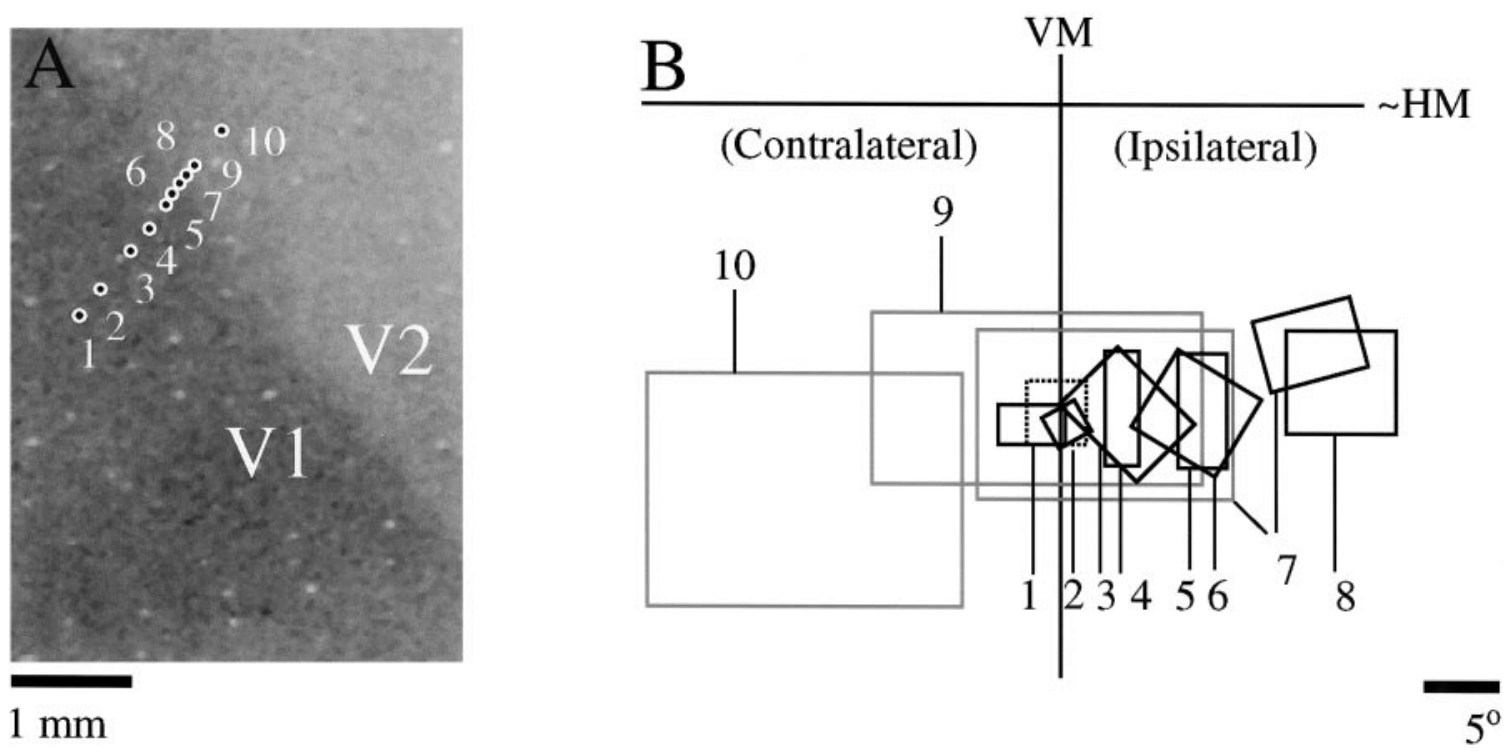

Figure 5. Physiological confirmation of the ipsilateral visual field representation (animal TS9756). $A$, Location of 10 recording sites relative to a Nissl-stained section cut tangentially through layer 4; the darkly stained region of the tissue corresponds to V1. $B$, Multiunit receptive field plots for the recording locations shown in $A$. For site 1 the contralateral (thick black line) and ipsilateral eye (dashed line) receptive fields were determined separately to confirm proper eye alignment. Receptive fields for all other sites were plotted using the contralateral eye only. Receptive fields for sites that appeared to be in V1 are shown in black, and receptive fields for sites that appeared to be in V2 are shown in gray. At site 7, recordings were made at two different depths, and receptive fields from both depths are shown. Based on these recordings, the V1/V2 border appeared to be near sites 7 and 8 , which corresponds to the position of the border on the Nissl-stained section.

Errors in alignment between sections were small, usually less than the diameter of the radial blood vessels.

At the conclusion of the alignment process, we created overlays by replacing the reference image with the optical imaging data and the radial vessel plot with the plots of injection sites and bead-labeled cells. In Figure $6, C$ and $D$, two overlays are shown for the same case that is illustrated in Figure 6, $A$ and $B$. The red bead injections were made into a region of cortex that was activated by a stimulus centered $8^{\circ}$ to the right of the VM (Fig. $6 C$ ). Most of the red bead-labeled cells in the left hemisphere were found in an area that was activated by the same stimulus. The green bead injection sites were made into a region that was activated by a stimulus centered on the VM (Fig. 6D). The green bead-labeled cells in the left hemisphere were found in a more lateral position than those labeled with red beads; this region was activated by the stimulus that was centered on the VM and not by the stimulus that was centered $8^{\circ}$ to the right of the VM. This experiment confirms that callosal connections tend to link sites in the two hemispheres that represent the same region of visual space.

Two overlays from an experiment in which we performed optical imaging and then made bead injections into an area of cortex representing part of the contralateral visual field are shown in Figure $6, E$ and $F$. Three injections of both red and green beads were made in two lines that extended roughly along isoazimuth lines, as determined by optical imaging. Both sets of bead injections were made within an area of cortex that responded strongly to a stimulus placed $4^{\circ}$ right of the VM (Fig. $6 E$ ). Again, the majority of the bead-labeled cells lie over regions of right visual cortex that were also activated by this stimulus, indicating that there is at least a rough visuotopic correspondence between the sites linked by callosal connections. The precision of the correspondence can be appreciated in several ways. First, note that although the red and green bead-labeled cell distributions are overlapping, the green bead-labeled cells, which were labeled by a more medial set of injections in left cortex, occupy a more lateral position in right visual cortex. The direction and magnitude of this shift are precisely what we would expect if callosal connections link visuotopically corresponding points. Second, the red bead injections were made in an area of cortex that also responded when a stimulus was placed at the VM, whereas the green bead injections were made in an area that did not respond to this stimulus (Fig. 6F). Correspondingly, most of the red bead-labeled cells are found in the activated region of the right cortex, whereas most of the green bead-labeled cells lie lateral to this region (Fig. $6 F$ ). Finally, the distributions of labeled cells observed after injection of beads into the contralateral visual field representation (Fig. 6E,F) occupy a much less extensive region of cortex than those obtained from injection of beads into the ipsilateral visual field representation (Fig. 6C,D, red injections). This pattern correlates with the difference in magnification factor for the ipsilateral and contralateral visual field representations.

Another example of the visuotopic specificity of callosal connections comes from an experiment in which we made single injections of red and green beads that were separated by only 500 $\mu \mathrm{m}$ (Fig. 7). The bead injections made in this case were slightly larger than those made in the two cases shown so far, and they were located within the ipsilateral visual field representation of left visual cortex. These factors explain the larger size of the labeled cell distributions seen in this case (Fig. 7A). The injections were made at a location that had a preferred position of $\sim 2^{\circ}$ left of the VM and, as expected, the distribution of labeled cells in the right hemisphere is centered on a region of cortex that responded strongly to the same stimulus (Fig. 7A). In addition, although the red and green distributions are highly overlapping, they are slightly shifted in the correct direction to maintain visuotopic correspondence. 


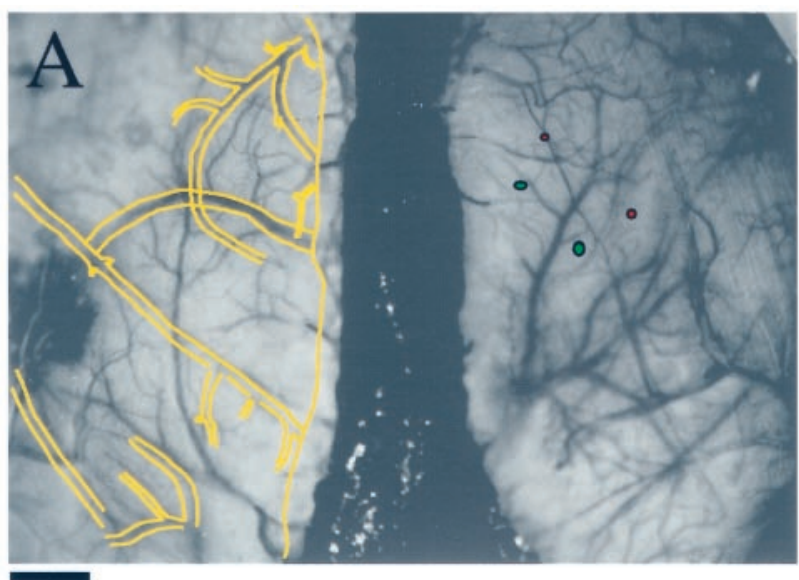

\section{$1 \mathrm{~mm}$}
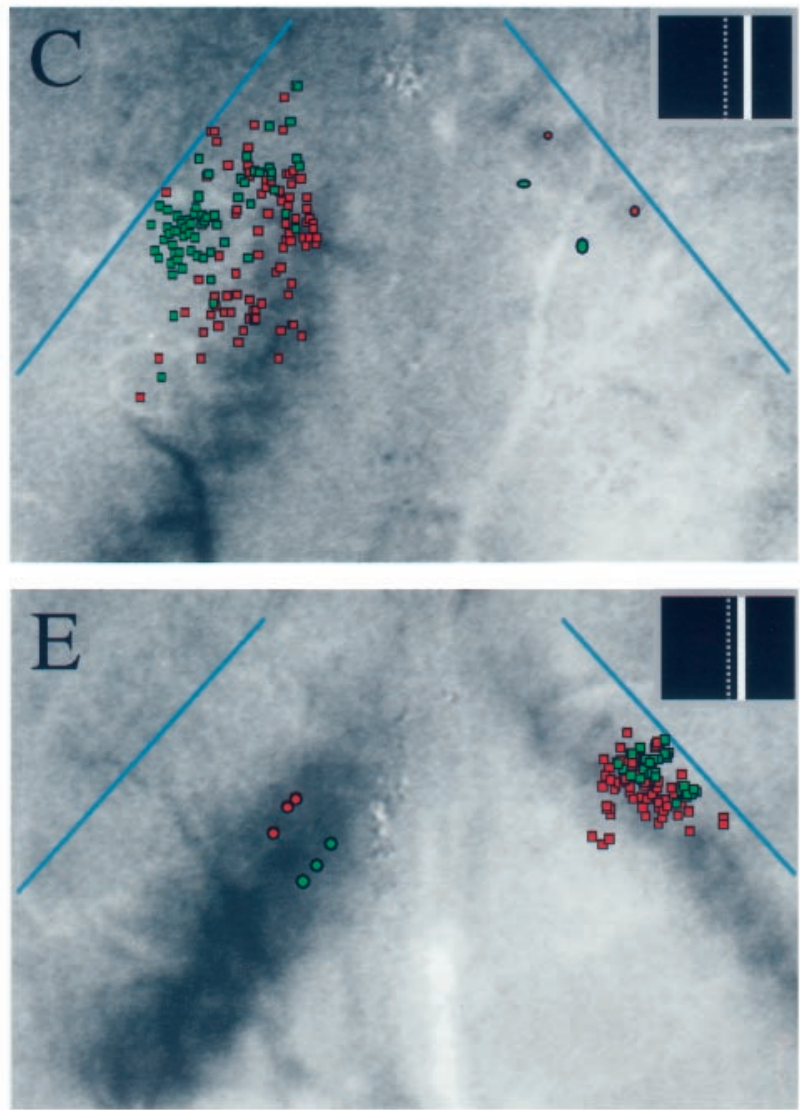

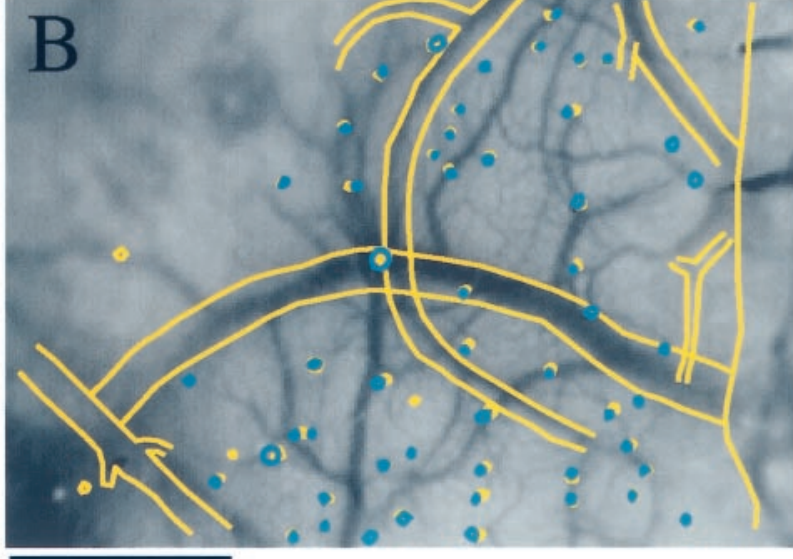

\section{$1 \mathrm{~mm}$}
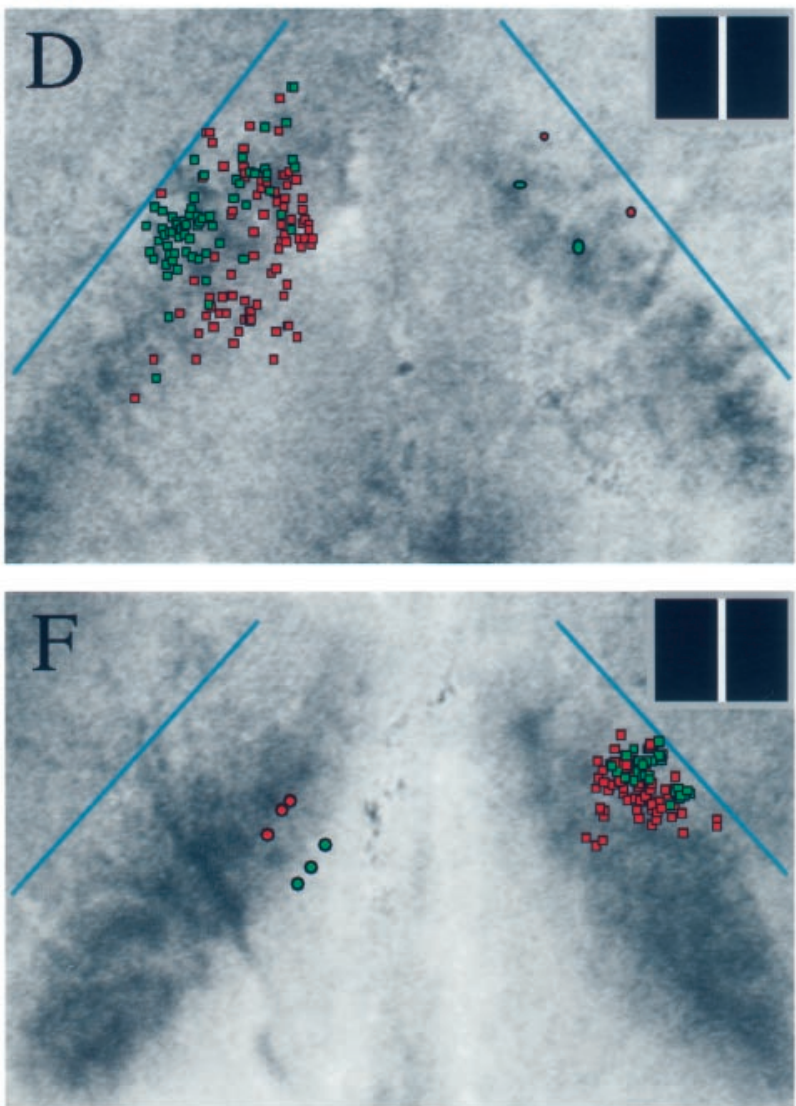

Figure 6. Visuotopic specificity of callosal connections. $A-D$, Results from an experiment in which optical imaging of intrinsic signals was combined with injections of fluorescent microspheres (animal TS9803). A, Alignment of the drawing of the first histological section (yellow overlay) to the reference image taken during optical imaging. The filled red and green ellipses indicate the location and approximate size of bead injections made in right visual cortex. Scale bar in $A$ applies to A and C-F. For details of alignment procedure, see Materials and Methods. $B$, Higher magnification view of the alignment of the first tissue section (yellow overlay) and a deeper tissue section (blue overlay) that contained bead-labeled cells. $C$, Location of bead injections ( filled red and green ellipses) and bead-labeled cells (red and green squares) shown over the cortical response to a stimulus placed $8^{\circ}$ right of the VM, as shown in the inset. The blue lines indicate the position of the V1/V2 border in each hemisphere. The red injections were made in a region of cortex that responded to this stimulus, and many of the red bead-labeled cells are found in an area of left visual cortex that also responded to this stimulus. $D$, The same bead injections and labeled cells as shown in $C$ shown over the response to a stimulus placed at the VM. The green bead injections were made in an area that responded to this stimulus, and green bead-labeled cells in the opposite hemisphere are found in a region that also responded to this stimulus. $E$, $F$, Visuotopic specificity of callosal connections originating in the ipsilateral visual field representation (animal TS9768). $E$, Bead injections and labeled cell distributions shown over the cortical response to a stimulus placed $4^{\circ}$ right of the VM, as shown in inset. F, Bead injections and labeled cells shown over the cortical response evoked by a stimulus located at the VM. 

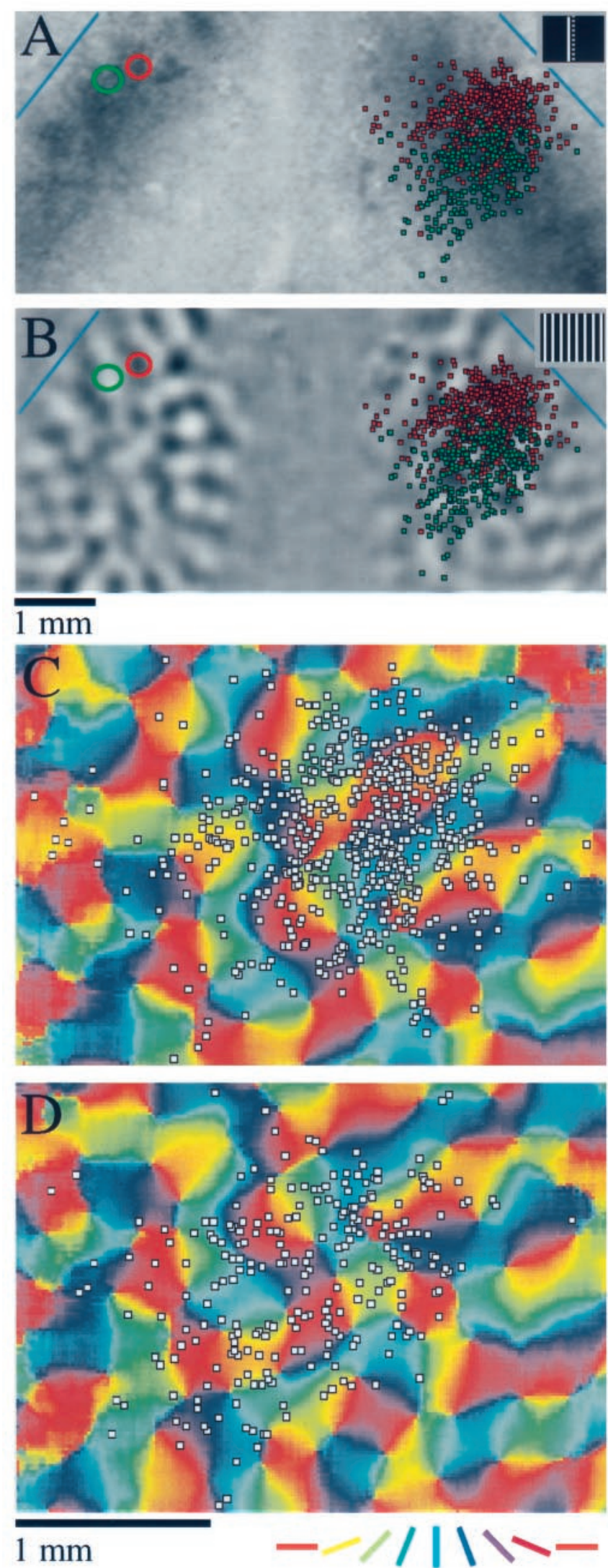

Figure 7. Visuotopic specificity and lack of modular specificity of callosal connections (animal TS9821). In this animal, one injection of red beads, indicated by the red circle, was placed in an area of cortex that had a vertical orientation preference and a position preference of $2^{\circ}$ left. One

\section{Modular and axial specificity of callosal connections}

Although the two injections in the case shown in Figure 7 were separated by a fairly small distance in cortex, they were centered in regions that had orthogonal orientation preferences, allowing us to assess the orientation specificity of the callosal label in the opposite hemisphere. Figure $7 B$ shows the location of the two injection sites and the labeled cell distributions over a difference image in which dark areas responded preferentially to a vertical stimulus. The red injection was located at a site that preferred vertical, and the green injection was located at a site that preferred horizontal. As illustrated in Figure $7 A$, the red and green cell distributions occupy highly overlapping regions of the opposite hemisphere. If callosal connections selectively link sites in the two hemispheres that have similar orientation preferences, we would expect each distribution to be arranged in a patchy or modular fashion. In addition, we would expect the red- and green-labeled cells to be largely segregated, with red cells found over regions with a vertical orientation preference, and green cells found over regions with a horizontal orientation preference. None of these expectations were met. As seen in Figure $7 B$, neither distribution is found selectively over the light or dark regions of the difference image. Furthermore, the red and green bead-labeled cells show little sign of a complementary distribution. The fine scale organization and orientation specificity of the distributions can be better examined by displaying each separately over an orientation preference map for the right hemisphere (Fig. 7C,D). Both populations of labeled cells appear rather evenly distributed, and each covers regions of cortex that include a wide range of orientation preferences.

The case shown in Figure 7 suggests that callosal connections lack specificity with respect to the map of orientation preference. However, it could be argued that the size of the injections may have been too great to reveal an underlying modular specificity. To verify that our methods are suitable for detecting the orientation specificity of cortical connections, we directly compared the specificity of intrinsic connections and callosal connections resulting from the same injection of beads in three animals. One of these experiments is shown in Figure 8. A single injection of red beads was made in the left hemisphere in an area of cortex that had a preferred orientation of $\sim 60^{\circ}$. In Figure $8 A$, the resulting distribution of labeled cells is shown over a difference image in which dark areas responded preferentially to a $45^{\circ}$ stimulus. The labeled cells in left cortex extend for several millimeters rostral and caudal of the injection site, and are found mostly over dark

\section{$\leftarrow$}

injection of green beads, indicated by the green circle, was made at a slightly more lateral position that had a horizontal orientation preference and a position preference of $4^{\circ}$ left. As in Figure 6, red bead-labeled cells are indicated by red squares, green bead-labeled cells are indicated by green squares, and blue lines indicate the position of the V1/V2 border. $A$, Bead injections and labeled cell distributions are shown over the cortical response to a stimulus placed $2^{\circ}$ left of the VM. $B$, Bead injections and labeled cell distributions shown over a difference image in which dark areas of the image indicate areas of cortex that responded preferentially to a vertical grating. Although the red injection was made in a vertical domain, and the green injection was made in a horizontal domain, the labeled cell distributions show no orientation specificity in the opposite hemisphere. Scale bar in $B$ applies to $A$ and $B$. $C$, Distribution of red bead-labeled cells, now shown as white squares, over an orientation preference image for part of the right hemisphere. $D$, Distribution of green bead-labeled cells over an orientation preference image for part of the right hemisphere. The scale bar and color key in $D$ each apply to both $C$ and $D$. 

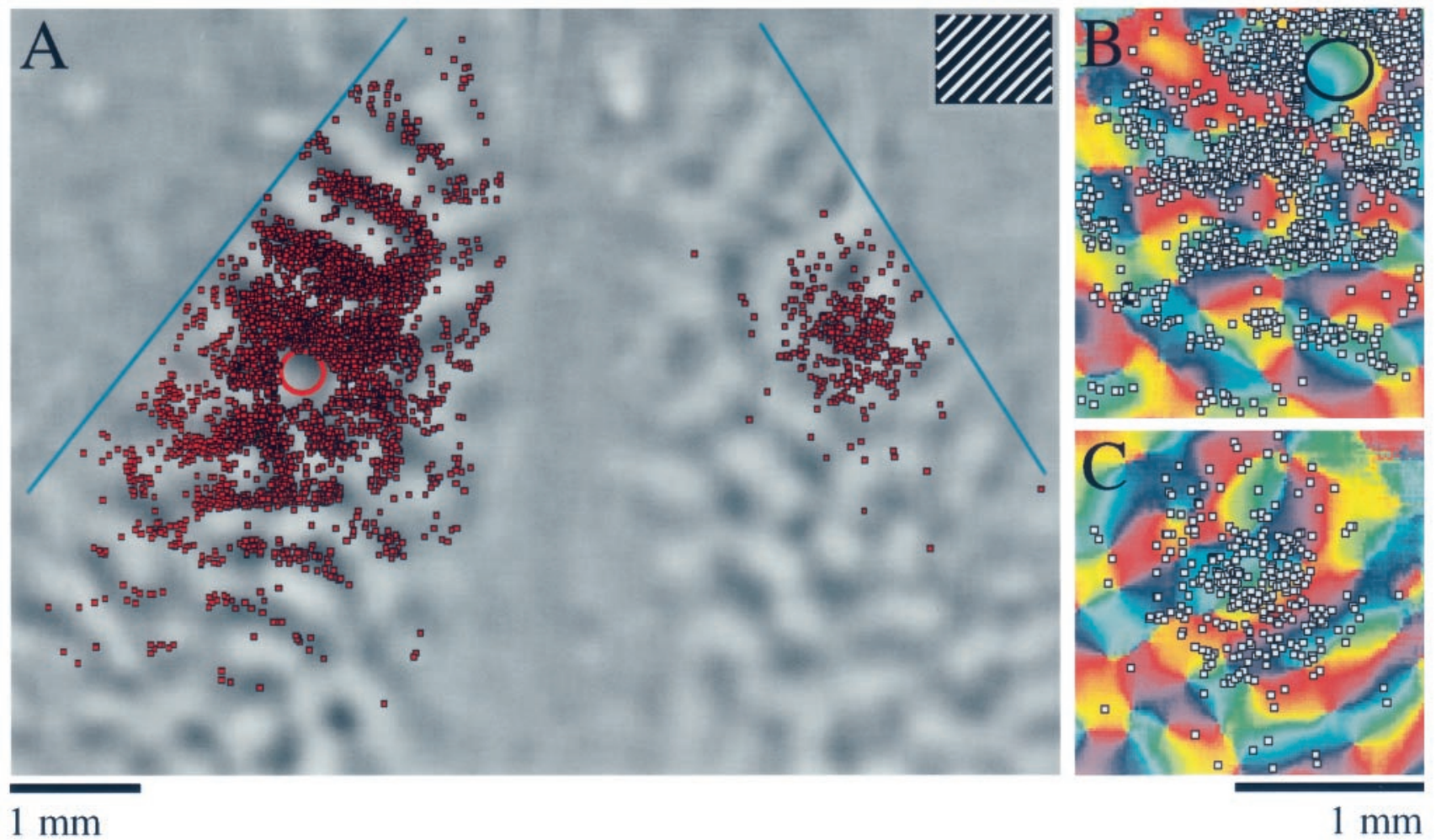

Figure 8. Comparison of modular specificity of intrinsic and callosal connections in a single animal (animal TS9824). A single injection of red beads was made in this animal in an area of the left cortex that had an orientation preference of near $60^{\circ}$, and the labeled cells in both hemispheres were plotted. $A$, Injection and labeled cells shown over an orientation difference image in which dark areas indicate regions of cortex that responded preferentially to a $45^{\circ}$ grating. The red circle indicates the limits of the injection site, and red squares indicate red bead-labeled cells. Labeled cells are found in a distribution that is elongated along roughly the rostrocaudal axis, corresponding to the preferred orientation of the injection site, and are found preferentially over dark areas of the difference image in left cortex. Labeled cells in the opposite hemisphere are found in a more restricted and symmetrically shaped distribution and show no preference for dark or light domains. $B$, Distribution of labeled cells found in the left cortex shown over an orientation preference map for that hemisphere. Labeled cells tend to lie over blue and green regions of the orientation preference map, indicating a preference for sites with orientation preferences between 45 and $90^{\circ} . C$, Distribution of labeled cells found in the right hemisphere displayed over an orientation preference map for that hemisphere. Labeled cells show no preference for a particular orientation. Orientation preference is color-coded according to key shown in Figure 7. Scale bar in $C$ applies to $B$ and $C$.

areas of the difference image, indicating modular specificity related to orientation preference. The labeled cells in the right hemisphere, on the other hand, are confined to a smaller area of cortex and show no obvious modularity. The same distributions are shown over the orientation preference map for this case in Figure $8, B$ and $C$. The intrinsic connections exhibit some variability near the injection site, but are found preferentially over blue-green areas of the map at longer distances (Fig. 8B). Few cells are found over the red and purple regions of the map, representing sites with an orientation preference near horizontal. Cells in the opposite hemisphere, on the other hand, appear evenly distributed with respect to the orientation preference map (Fig. 8C).

To quantify the specificity of the labeled intrinsic connections $(n=3)$ and of labeled callosal connections $(n=6)$, we created cell tuning curves by calculating the percentage of the total number of cells found in different orientation preference bins relative to the preferred orientation of the injection site. Figure $9 A$ shows that each of the three intrinsic cases showed a strong bias toward connecting sites with similar orientation preference to the injection site, and the group average shows the same bias. Although two of the tuning curves for individual callosal distributions shown in Figure $9 B$ do seem to show a peak near the preferred orientation of the injection site, the overall tuning of the callosal distributions was much more erratic, and the group average is close to what would be expected for flat, or random, tuning.

We compared the specificity of callosal connections and intrinsic connections by quantifying the percentage of labeled cells found in areas with similar $\left( \pm 35^{\circ}\right)$ orientation preference to the injection site (Fig. 9C). Also included in this comparison is the specificity of intrinsic connections as measured in our previous study using biocytin as an anterograde tracer and counting the number of labeled boutons (Bosking et al., 1997). The two measures of the specificity of intrinsic connections are in good agreement and exhibit a large difference from the percentage of isoorientation contacts expected for an even distribution. Callosal connections, on the other hand, show a much smaller, if any, bias toward linking sites with the same orientation preference.

Although we did not attempt to quantify the axial specificity of our labeled cell distributions, callosal connections do not exhibit the orientation-specific elongation that is characteristic of longrange intrinsic connections. Neurons in layer $2 / 3$ give rise to horizontal connections that extend for greater distances, and make more contacts, along an axis in the map of visual space that corresponds to their preferred orientation (Bosking et al., 1997; Fig. 9). This type of axial specificity is apparent in the case shown in Figure 8, in which a single injection of beads led to an 

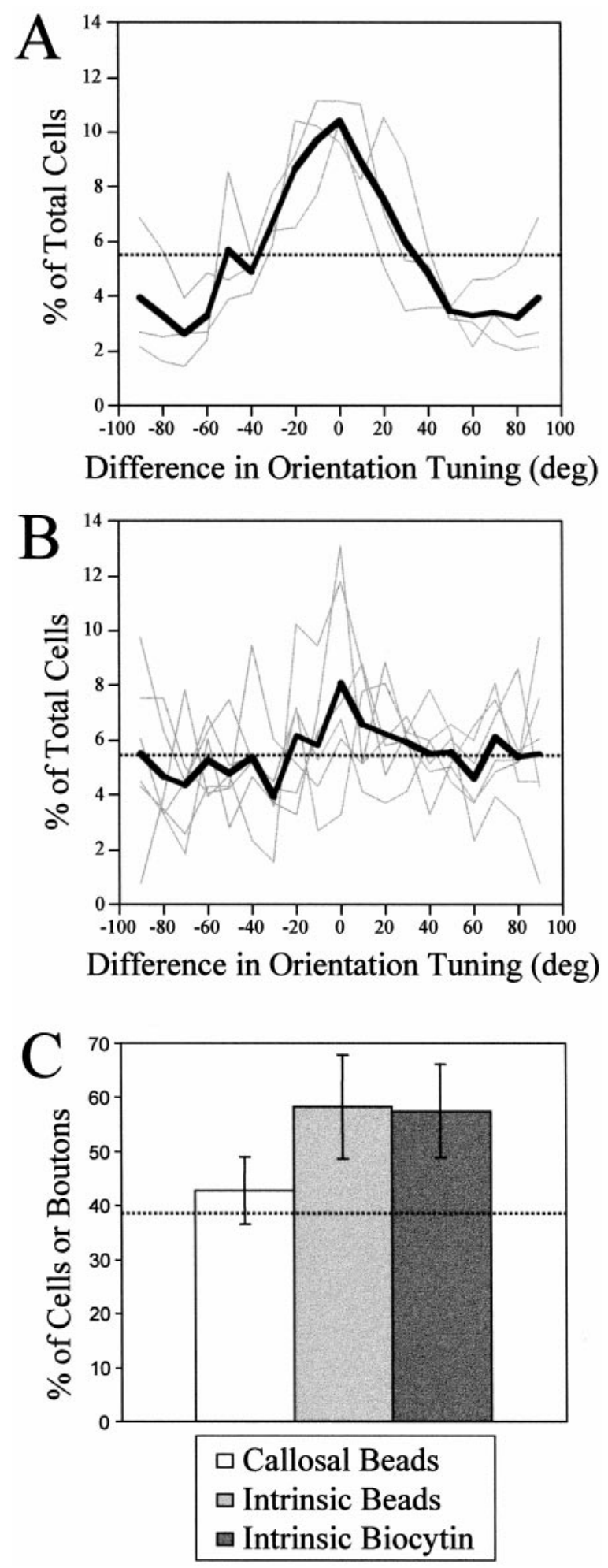

Figure 9. Quantification of orientation specificity of labeled cell distributions. A, Tuning curves for intrinsic cell distributions. Each curve shows the percentage of total cells found in different orientation preference bins relative to the preferred orientation of the injection site. Cells shown in the $0^{\circ}$ bin had the same orientation preference as the injection site, those shown in the $90^{\circ}$ bin had an orientation preference orthogonal to the injection site. Only cells located further than $500 \mu \mathrm{m}$ from the injection site were considered for this analysis. The three gray curves show the tuning for the three individual cases we examined; the thick black line shows the group average. The dotted line indicates the percentage of the total labeled cells that would be expected in each bin for an even elongated distribution of cells within the same hemisphere. The distribution is elongated along an axis that is slightly shifted from the vertical axis in the map of visual space, corresponding to the $60^{\circ}$ orientation preference of the injection site. A much smaller, and more symmetrically shaped, distribution of cells is observed in the opposite hemisphere. In general, the callosal cell distributions we observed occupied a much smaller extent of cortex, and when injections were made near the representation of the VM, the labeled cell distributions were roughly symmetric in shape in the opposite hemisphere (Figs. $6 C, D$, green-labeled distributions, $8)$. When asymmetric distributions of labeled cells were seen in the opposite hemisphere, the asymmetry was either elongation or compression along an axis roughly orthogonal to the V1/V2 border. This anisotropy in connections is likely to be explained by the compression of the ipsilateral visual field representation along the horizontal axis. Injections made in the ipsilateral visual field representation led to labeled cell distributions in the contralateral visual field representation of the opposite hemisphere that were expanded along this axis; i.e., orthogonal to the V1/V2 border (Figs. 6C,D, red-labeled cell distributions, 7). Injections in the contralateral visual field representation, on the other hand, led to labeled cell distributions in the ipsilateral visual field representation of the opposite hemisphere that were compressed along this axis (Fig. 6E,F, green-labeled distributions).

\section{DISCUSSION}

The results presented here provide several new insights into the functional organization of callosal connections. First, these connections are organized in a highly topographic manner, linking together sites in the two hemispheres that respond to the same region of visual space. Second, callosal connections are not limited to regions of $\mathrm{V} 1$ that represent the VM: because of the presence of a substantial ipsilateral visual field representation, callosal connections may exert an influence over almost the entire binocular visual field representation of tree shrew V1. Finally, unlike other systems of cortical connections, callosal connections terminate without respect for the modular arrangement of orientation-selective neurons. Combined, these results provide a more complete picture of the functional architecture that forms the substrate for processing of information from central visual space and suggest ways in which the role of callosal connections may be distinguished from that of other cortical connections.

\section{Bilateral representation of central visual space}

The presence of an ipsilateral visual field representation is not unique to the tree shrew. Although it is most frequently associated with albino or pigment-deficient animals (Hubel and Wiesel, 1971; Shatz, 1977a; Shatz and LeVay, 1979; Ault et al., 1995), an ipsilateral visual field representation has been demonstrated in normally pigmented individuals from a variety of nonprimate

\section{$\leftarrow$}

distribution $(5.56 \%)$. B, Tuning curves for callosal cell distributions. The six gray curves show the tuning for the six individual cases we examined; the thick black line shows the group average. $C$, Comparison of specificity of callosal and intrinsic connections. Each bar indicates the percentage of the total number of cells or boutons that are found in areas that have similar $\left( \pm 35^{\circ}\right)$ orientation preference to the injection site $($ mean $\pm \mathrm{SD})$. The percentages reported for specificity of intrinsic and callosal connections measured by retrograde labeling with beads are from the present results, the percentage reported for specificity of intrinsic connections measured by anterograde labeling with biocytin comes from a previous report (Bosking et al., 1997). The dashed line indicates the percentage of cells or boutons that would be expected for an even distribution (38.9\%). 

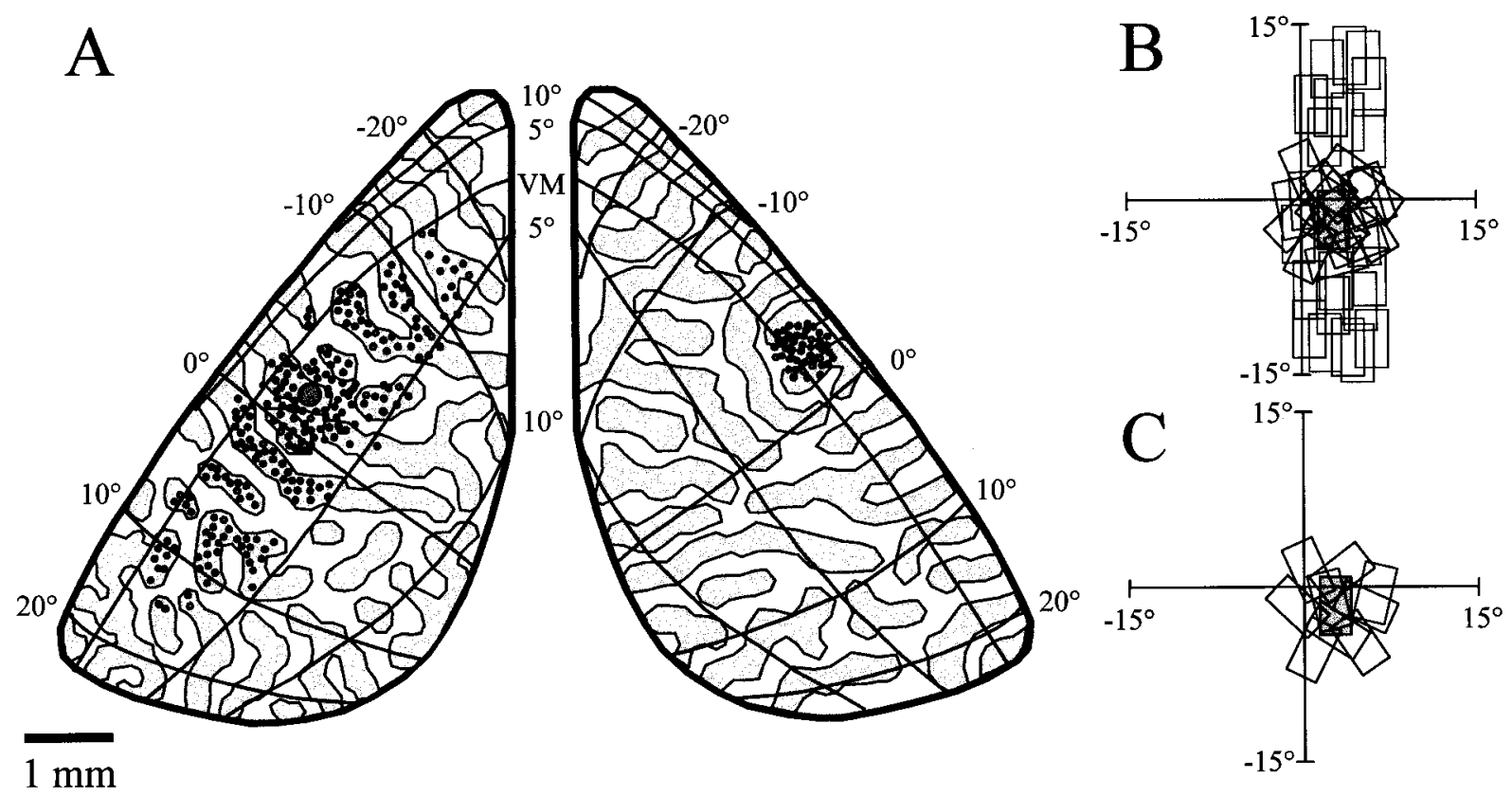

Figure 10. Summary of the bilateral representation of visual space and the specificity of horizontal and callosal connections in the tree shrew. $A$, Map of visual space modified from one first presented by Kaas et al. (1972) to reflect the ipsilateral visual field representation revealed by optical imaging. The dorsal portion of V1 is shown for both left and right visual cortex. Rostral is toward the top of the page. The thick black lines form the border of $\mathrm{V} 1$, and the thin black lines in each hemisphere indicate isoelevation and isoazimuth lines within the map of visual space. The dark gray and light gray regions beneath the map of visual space simulate vertical and horizontal orientation preference domains, respectively. The small dark gray circles in each hemisphere indicate the location of cells that would be likely to provide input to the site in left cortex indicated by the larger gray circle. The intrinsic inputs, in left cortex, are nonspecific at short distances, but at longer distances they originate mainly from sites along the vertical axis in the map of visual space and whose orientation preference is near vertical. The callosal input originates from a much smaller region of the opposite hemisphere and comes from regions that have both vertical and horizontal orientation preference. $B$, Visual field depiction of input received via horizontal connections. The dark gray rectangle in the background indicates the receptive field of a cell located where the large gray circle is found in the left cortex. The open rectangles indicate the receptive field locations of the cells that would provide input to this cell via horizontal connections. These inputs have receptive fields that span a total distance of $\sim 30^{\circ}$ of visual space. The cell receives input from many other cells with overlapping receptive fields that can be of any orientation and from other cells that have nonoverlapping receptive fields and whose receptive fields are displaced along a vertical line in visual space and have an orientation preference near $90^{\circ}$. C, Visual field depiction of input received via callosal connections. The inputs received from callosal connections have receptive fields confined to a much smaller region of visual space, and they include a wide range of orientation preferences.

species including cat, ferret, ground squirrel, rabbit, opossum, hamster, and sheep (Clarke and Whitteridge, 1976; Tiao and Blakemore, 1976; Hughes and Vaney, 1982; Whitteridge and Clarke, 1982; Pettigrew et al., 1984; Volchan et al., 1988; Payne, 1990; Sereno et al., 1991; White et al., 1999). The presence of an ipsilateral visual field representation appears to be linked to the decussation pattern of ganglion cells in the retina, specifically the fact that the crossed projection is not limited to the nasal retina, but extends with reduced density across the representation of the VM and into the temporal retina (Illing and Wassle, 1981; Pettigrew et al., 1984; Morgan et al., 1987; Baker and Reese, 1993; Ault et al., 1995; Jeffery et al., 1998). Indeed, the characteristics of the crossed temporal projection help to explain some of the unique features of the ipsilateral visual field representation. For example, the compression (low magnification) of the ipsilateral visual field representation relative to that of the contralateral visual field representation is consistent with the difference in the density of ganglion cells that supplies the two representations. Likewise, the variation in the extent of the ipsilateral visual field representation with eccentricity is consistent with the variation in the extent of the crossed temporal projection. In the tree shrew, for example, the ipsilateral visual field representation is smallest near the representation of the horizontal meridian and expanded in the lower visual field. Correspondingly, the density of the crossed temporal projection is lowest near the area centralis and highest in regions of the retina that support the upper and lower visual field (Jeffery et al., 1998). We were unable to determine whether the ipsilateral visual field representation is also expanded for the upper visual field in the tree shrew, because this region of cortex is not accessible for imaging. In the cat, however, the ipsilateral visual field representation is expanded in both the upper and lower visual field (Payne, 1990).

\section{Visuotopic arrangement of callosal connections}

Our results show that callosal connections are arranged in a highly specific manner with respect to the bilateral representation of visual space within V1. Callosal connections extend for up to $15^{\circ}$ into the contralateral visual field representation in V1, a figure that matches the extent of the ipsilateral visual field representation. Moreover, these connections are arranged in a topographic fashion that links visuotopically corresponding sites in the two hemispheres.

The best evidence for visuotopic correspondence comes from experiments in which we combined imaging for position with injections of retrograde tracers. Injections into an active zone in one hemisphere always labeled cells in the other hemisphere that were in zones activated by the same visual stimulus. The alignment was not perfect in every experiment, inasmuch as the labeled cells were not always centered on the area of activation, but it seems likely that these slight misalignments are attributable 
to the small $\left(1-2^{\circ}\right)$ errors in eye alignment that we detected with multiunit physiological recordings. Further support for visuotopic precision in callosal connections comes from experiments in which we injected two different tracers at nearby locations in cortex. Labeled cells were always found in overlapping, but precisely shifted, distributions in the opposite hemisphere, and the magnitude and direction of the shift was consistent with the demonstrated visuotopy. In addition, injections of tracers into the ipsilateral visual field representation always labeled a large patch of cells in the other hemisphere, whereas injections into the contralateral visual field representation labeled a comparatively small patch (compare Fig. $6 C, D$ and Fig. 7 to Fig. 6E,F). These differences are consistent with the difference in magnification factor of the ipsilateral and contralateral visual field representations. Overall, these results provide strong support for the view that callosal connections are centered around visuotopic correspondence. It is also clear, however, that these connections provide input from a moderate-sized region surrounding exact correspondence. Thus, a particular site in one hemisphere would receive input via callosal connections primarily from sites in the other hemisphere that had overlapping receptive fields, but also from sites whose receptive fields could be displaced by as much as the receptive field diameter of a layer $2 / 3$ neuron $\left(5^{\circ}\right)$.

The present findings were foreshadowed by earlier anatomical studies in the tree shrew showing that callosal projections extend several millimeters away from the V1/V2 border and that they are organized in a non-mirror-symmetric manner such that sites near the V1/V2 border are connected with sites displaced from the V1/V2 border in the opposite hemisphere (Sesma et al., 1984; Cusick et al., 1985; Pritzel et al., 1988; Kretz and Rager, 1990; Lyon et al., 1998). However, without the knowledge of the ipsilateral visual field representation within V1, the full significance of this pattern was not appreciated. A similar anatomical study in the cat, where an ipsilateral visual field representation had been identified (Whitteridge and Clarke, 1982; Payne, 1990, 1991; Payne and Siwek, 1991), proposed at least a rough visuotopic correspondence for callosal connections (Olavarria, 1996a). However, these experiments did not combine anatomy and physiology in the same animal and, as a result, the exact relationship between the visual field locations of the injection sites and the distribution of labeled cells could not be assessed.

The visuotopic organization demonstrated here challenges the classical view that callosal connections are concerned exclusively with the visual midline and are the sole source of ipsilateral visual field input for cells whose receptive fields straddle the visual midline (Hubel and Wiesel, 1967; Berlucchi and Rizzolatti, 1968). Instead, callosal connections appear to provide visuotopically corresponding inputs for neurons whose receptive fields are well removed from the $\mathrm{VM}$ as well as for neurons whose receptive fields span the visual midline. As noted by others, there is no need for callosal connections to extend cortical receptive fields across the midline because information from the ipsilateral visual field is already present in the pattern of ganglion cell projections to the lateral geniculate nucleus (Blakemore et al., 1983). Thus, callosal connections are arranged in register with lateral geniculate terminations, i.e., they link sites in the two hemispheres that receive lateral geniculate inputs representing the same region of visual space. How the inputs from these two sources contribute to the responses of individual cortical neurons, however, remains unclear. Although the density of projection suggests that geniculatederived inputs are likely to dominate the responses of layer $2 / 3$ neurons, under certain circumstances callosal connections appear to be capable of supporting the selective responses of neurons in lieu of LGN inputs (Choudhury et al., 1965; Berlucchi and Rizzolatti, 1968; Lepore and Guillemot, 1982; Blakemore et al., 1983).

The demonstration that callosal connections provide topographically precise connections for regions of visual space that are represented bilaterally also offers a simple explanation for differences among species in the cortical extent of the callosal connections: species differences in the amount of visual space that is represented bilaterally. The relatively restricted extent of callosal connections in many primates and the widespread distribution of callosal connections in most nonprimates and in abnormally pigmented animals parallels the extent of the crossed inputs from the temporal retina (Shatz, 1977b,c; Swadlow et al., 1978; Cusick et al., 1984, 1985; Sesma et al., 1984; Kennedy et al., 1986; Pritzel et al., 1988; Kretz and Rager, 1990; Payne, 1991; Payne and Siwek, 1991; Grigonis et al., 1992; Ault et al., 1995). It follows, then, that the visuotopic precision demonstrated here is a feature of callosal connections in most mammals, regardless of the extent of the bilateral representation.

\section{Lack of orientation specificity to the callosal projections}

Our results demonstrate that there is little orientation specificity to the distribution of callosal connections. Restricted injections of tracers into sites that produced a prominent patchy distribution of labeled cells in iso-orientation domains of the ipsilateral hemisphere resulted in a single patch of labeled cells in the contralateral hemisphere that displayed little sign of selectivity for the orientation preference of the cells at the injection site. This result is perhaps surprising based on the results of anatomical and physiological experiments performed in the cat.

In general, callosal connections in the cat have been regarded as terminating in an orientation-specific manner, and it is possible that there are differences between the organization of callosal connections in these two species. However, the evidence on this point is far from definitive. For example, the demonstration that callosal connections terminate in a patchy manner has led some investigators to suggest linkage between sites with similar orientation preference (Houzel et al., 1994). However, other studies demonstrate that the patchy distribution of callosal connections is related to other types of functional units such as ocular dominance domains or cytochrome oxidase blobs (Boyd and Matsubara, 1994; Olavarria, 1996b). Only one study has addressed the relation between callosal connections and orientation domains defined by uptake of 2-deoxyglucose, and the authors concluded that callosal connections were biased for sites with the same preferred orientation (Schmidt et al., 1997). However, this study was based on the analysis of connections in strabismic animals, and it is not clear whether decorrelating the activity in the two eyes alters the normal relationship between callosal connections and maps of orientation preference. Interpretation of this result is also made difficult by the fact that the analysis was based on experiments that combined 2-DG labeling for both ocular dominance and orientation, and it is not clear how much of the specificity can be attributed to orientation alone. Clearly, this issue warrants further study in normal animals.

\section{Functional architecture of callosal interactions}

Our results demonstrate that callosal connections are organized according to principles that differ significantly from long-range horizontal connections (Fig. 10). Both the modular and axial 
specificity that are such striking features of long-range horizontal connections are largely absent from callosal connections. Indeed, the primary factor constraining the distribution of callosal connections appears to be visuotopic correspondence. In this respect, callosal connections seem similar to local intrinsic connectionsconnections that extend in a symmetrical fashion for a radius of $\sim 500 \mu \mathrm{m}$ and contact neurons that have largely overlapping receptive fields and may exhibit a wide range of preferred orientations. By coordinating the activity in the two hemispheres in a way that preserves nearest neighbor relationships, callosal connections may best be viewed as elements of local circuits that operate within a single bilateral representation of visual space.

\section{REFERENCES}

Ault SJ, Leventhal AG, Vitek DJ, Creel DJ (1995) Abnormal ipsilateral visual field representation in areas 17 and 18 of hypopigmented cats. J Comp Neurol 354:181-192.

Baker GE, Reese BE (1993) Chiasmatic course of temporal retinal axons in the developing ferret. J Comp Neurol 330:95-104.

Berlucchi G, Rizzolatti G (1968) Binocularly driven neurons in visual cortex of split-chiasm cats. Science 159:308-310.

Blakemore C, Diao YC, Pu ML, Wang YK, Xiao YM (1983) Possible functions of the interhemispheric connexions between visual cortical areas in the cat. J Physiol (Lond) 337:331-349.

Blasdel GG (1992) Orientation selectivity, preference, and continuity in monkey striate cortex. J Neurosci 12:3139-3161.

Bonhoeffer T, Grinvald A (1991) Iso-orientation domains in cat visual cortex are arranged in pinwheel- like patterns. Nature 353:429-431.

Bonhoeffer T, Grinvald A (1993) The layout of iso-orientation domains in area 18 of cat visual cortex: optical imaging reveals a pinwheel-like organization. J Neurosci 13:4157-4180.

Bosking WH, Zhang Y, Schofield B, Fitzpatrick D (1997) Orientation selectivity and the arrangement of horizontal connections in tree shrew striate cortex. J Neurosci 17:2112-2127.

Boyd J, Matsubara J (1994) Tangential organization of callosal connectivity in the cat's visual cortex. J Comp Neurol 347:197-210.

Choudhury BP, Whitteridge D, Wilson ME (1965) The function of the callosal connections of the visual cortex. Q J Exp Physiol 50:214-219.

Clarke PG, Whitteridge D (1976) The cortical visual areas of the sheep. J Physiol (Lond) 256:497-508.

Cusick CG, Gould III HJ, Kaas JH (1984) Interhemispheric connections of visual cortex of owl monkeys (Aotus trivirgatus), marmosets (Callithrix jacchus), and galagos (Galago crassicaudatus). J Comp Neurol 230:311-336.

Cusick CG, MacAvoy MG, Kaas JH (1985) Interhemispheric connections of cortical sensory areas in tree shrews. J Comp Neurol 235:111-128.

Gilbert CD, Wiesel TN (1983) Clustered intrinsic connections in cat visual cortex. J Neurosci 3:1116-1133.

Grigonis AM, Rayos del Sol-Padua RB, Murphy EH (1992) Visual callosal projections in the adult ferret. Vis Neurosci 9:99-103.

Houzel JC, Milleret C, Innocenti G (1994) Morphology of callosal axons interconnecting areas 17 and 18 of the cat. Eur J Neurosci 6:898-917.

Hubel DH, Wiesel TN (1967) Cortical and callosal connections concerned with the vertical meridian of visual fields in the cat. J Neurophysiol 30:1561-1573.

Hubel DH, Wiesel TN (1971) Aberrant visual projections in the Siamese cat. J Physiol (Lond) 218:33-62.

Hughes A, Vaney DI (1982) The organization of binocular cortex in the primary visual area of the rabbit. J Comp Neurol 204:151-164.

Illing RB, Wassle H (1981) The retinal projection to the thalamus in the cat: a quantitative investigation and a comparison with the retinotectal pathway. J Comp Neurol 202:265-285.

Innocenti GM (1986) General organization of callosal connections in the cerebral cortex. In: Cerebral cortex, Vol 5, Sensory-motor areas and aspects of cortical connectivity (Jones EG, Peters A, eds), pp 291-353. New York: Plenum.

Jeffery G, Harman A, Flugge G (1998) First evidence of diversity in eutherian chiasmatic architecture: tree shrews, like marsupials, have spatially segregated crossed and uncrossed chiasmatic pathways. J Comp Neurol 390:183-193.

Kaas JH (1980) A comparative survey of visual cortex organization in mammals. In: Comparative neurology of the telencephalon (Ebbesson SOE, ed), pp 483-502. Bew Tirj: Plenum.
Kaas JH, Hall WC, Killackey H, Diamond IT (1972) Visual cortex of the tree shrew (Tupaia glis): architectonic subdivisions and representations of the visual field. Brain Res 42:491-496.

Kennedy H, Dehay C, Bullier J (1986) Organization of the callosal connections of visual areas V1 and V2 in the macaque monkey. J Comp Neurol 247:398-415.

Kennedy H, Meissirel C, Dehay C (1991) Callosal pathways in primates and their compliancy to general rules governing the organization of corticocortical connectivity. In: Vision and visual dysfunction, Vol 3 (Cronly-Dillon J, gen ed), Neuroanatomy of the visual pathways and their development (Dreher B, Robinson S, eds), pp 324-359. London: Macmillan.

Kretz R, Rager G (1990) Reciprocal heterotopic callosal connections between the two striate areas in Tupaia. Exp Brain Res 82:271-278.

Lepore F, Guillemot JP (1982) Visual receptive field properties of cells innervated through the corpus callosum in the cat. Exp Brain Res 46:413-424.

Lyon DC, Jain N, Kaas JH (1998) Cortical connections of striate and extrastriate visual areas in tree shrews. J Comp Neurol 401:109-128.

Morgan JE, Henderson Z, Thompson ID (1987) Retinal decussation patterns in pigmented and albino ferrets. Neuroscience 20:519-535.

Olavarria JF (1996a) Non-mirror-symmetric patterns of callosal linkages in areas 17 and 18 in cat visual cortex. J Comp Neurol 366:643-655.

Olavarria JF (1996b) Visual callosal fibers link cortical columns dominated by the same eye in the cat. Soc Neurosci Abstr 22:491.

Payne BR (1990) Representation of the ipsilateral visual field in the transition zone between areas 17 and 18 of the cat's cerebral cortex. Vis Neurosci 4:445-474.

Payne BR (1991) Visual-field map in the transcallosal sending zone of area 17 in the cat. Vis Neurosci 7:201-219.

Payne BR, Siwek DF (1991) Visual-field map in the callosal recipient zone at the border between areas 17 and 18 in the cat. Vis Neurosci 7:221-236.

Pettigrew JD, Ramachandran VS, Bravo H (1984) Some neural connections subserving binocular vision in ungulates. Brain Behav Evol 24:65-93.

Press WH (1992) Numerical recipes in C: the art of scientific computing, Ed 2, pp 326-330. Cambridge: Cambridge UP.

Pritzel M, Kretz R, Rager G (1988) Callosal projections between areas 17 in the adult tree shrew (Tupaia belangeri). Exp Brain Res 72:481-493.

Rockland KS, Lund JS (1982) Widespread periodic intrinsic connections in the tree shrew visual cortex. Science 215:1532-1534.

Schmidt KE, Kim DS, Singer W, Bonhoeffer T, Lowel S (1997) Functional specificity of long-range intrinsic and interhemispheric connections in the visual cortex of strabismic cats. J Neurosci 17:5480-5492.

Sereno MI, Rodman HR, Karten HJ (1991) Organization of visual cortex in the California ground squirrel. Soc Neurosci Abstr 17:844.

Sesma MA, Casagrande VA, Kaas JH (1984) Cortical connections of area 17 in tree shrews. J Comp Neurol 230:337-351.

Shatz C (1977a) A comparison of visual pathways in Boston and Midwestern Siamese cats. J Comp Neurol 171:205-228.

Shatz C (1977b) Abnormal interhemispheric connections in the visual system of Boston Siamese cats: a physiological study. J Comp Neurol 171:229-245.

Shatz CJ (1977c) Anatomy of interhemispheric connections in the visual system of Boston Siamese and ordinary cats. J Comp Neurol 173:497-518.

Shatz CJ, LeVay S (1979) Siamese cat: altered connections of visual cortex. Science 204:328-330.

Swadlow HA, Weyand TG, Waxman SG (1978) The cells of origin of the corpus callosum in rabbit visual cortex. Brain Res 156:129-134.

Tiao YC, Blakemore C (1976) Functional organization in the visual cortex of the golden hamster. J Comp Neurol 168:459-481.

Volchan E, Bernardes RF, Rocha-Miranda CE, Gleiser L, Gawryszewski LG (1988) The ipsilateral field representation in the striate cortex of the opossum. Exp Brain Res 73:297-304.

Weliky M, Kandler K, Fitzpatrick D, Katz LC (1995) Patterns of excitation and inhibition evoked by horizontal connections in visual cortex share a common relationship to orientation columns. Neuron 15:541-552.

White LE, Bosking WH, Williams SM, Fitzpatrick D (1999) Maps of central visual space in ferret V1 and V2 lack matching inputs from the two eyes. J Neurosci 19:7089-7099.

Whitteridge D, Clarke PG (1982) Ipsilateral visual field represented in the cat's visual cortex. Neuroscience 7:1855-1860. 